\title{
CWENO Finite-Volume Interface Capturing Schemes for Multicomponent Flows Using Unstructured Meshes
}

\author{
Panagiotis Tsoutsanis ${ }^{1}$ (D) Ebenezer Mayowa Adebayo ${ }^{1} \cdot$ Adrian Carriba Merino $^{1}$. \\ Agustin Perez Arjona ${ }^{1} \cdot$ Martin Skote ${ }^{1}$
}

Received: 11 March 2021 / Revised: 13 September 2021 / Accepted: 29 September 2021 /

Published online: 9 November 2021

(c) The Author(s) 2021

\begin{abstract}
In this paper we extend the application of unstructured high-order finite-volume centralweighted essentially non-oscillatory (CWENO) schemes to multicomponent flows using the interface capturing paradigm. The developed method achieves high-order accurate solution in smooth regions, while providing oscillation free solutions at discontinuous regions. The schemes are inherently compact in the sense that the central stencils employed are as compact as possible, and that the directional stencils are reduced in size, therefore simplifying their implementation. Several parameters that influence the performance of the schemes are investigated, such as reconstruction variables and their reconstruction order. The performance of the schemes is assessed under a series of stringent test problems consisting of various combinations of gases and liquids, and compared against analytical solutions, computational and experimental results available in the literature. The results obtained demonstrate the robustness of the new schemes for several applications, as well as their limitations within the present interface-capturing implementation.
\end{abstract}

Keywords CWENO · Unstructured · Finite-volume · Multispecies

\section{Introduction}

Multi-component multi-phase compressible flows are encountered in several scientific fields and due to the complexity of the interaction of different states of matter a detailed understanding of them is required for many applications. The choice of schemes to simulate these interactions are far neither unique nor obvious, due to several factors such as the level of fidelity, the computational resources, and due to the mechanism of the interface that separates two fluids (e.g. liquid and gas), since the thermodynamic properties of the interface might be different from the two separate states, that can obey different equation of state (EOS). Most of the schemes in an Eulerian framework belong to the class of sharp inter-

$凶$ Panagiotis Tsoutsanis

panagiotis.tsoutsanis@cranfield.ac.uk

1 School of Aerospace, Transport and Manufacturing, Cranfield University, Cranfield MK43 0AL, UK 
face type methods (SIM) or the class of diffuse interface methods (DIM). The SIM class of methods treat the material interface between the fluids as infinitely thin, or sharp and several successful methods have been developed including the volume-of-fluid [28], the front-tracking [55], and the level-set methods [40]. The DIM class of methods is treating the interface as a non-zero thickness based on the idea originally formulated by Rayleigh [45] and van der Waals [69] who developed gradient theories for predicting the thickness of the interfaces based on thermodynamic principles. The DIM allow artificially the two states to develop some mixing, and therefore a thermodynamic state of this mixture is required as the interface evolves. Among the common DIM models are the five-equation model of Kapila's et al. [35], which is tailored for two-phase immiscible non-reacting flows which was also adopted by Allaire's et al. [1]. The complete Baer-Nunziato's [6] seven equation model that includes non-equilibrium effects and the unified hyperbolic formulation of GodunovPeshkov-Romenski (GPR model) [24,42] that can treat traditional fluid and solid problems within one framework which is the ultimate goal of several highly sophisticated and elegant advanced diffuse interface models [8,14,22,23,29,38,70,71].

In the present study the five-equation model of Kapila et al. [35] is employed in the form adopted by Allaire et al. [1] where an isobaric closure law is deployed that can simulate two fluids with arbitrary EOSs. This form has been extended by numerous researchers to include viscosity, capillary effects, and has been also extended to unstructured meshes by Chiapolino et al. [11], Price et al. [43], Faucher et al. [21] and Cheng et al. [10]. The simplified and more compact five-equation model of Allaire et al. has found many applications as listed in $[10,11,13,21,32,36,43,47,72,73]$.

The hyperbolic character of the PDEs involved in this context, the simplicity of this type of DIM and their ease of implementation are the primary reasons for the selection of this five-equation model. For engineering applications unstructured meshes offer significant advantages when dealing with optimisation of complicated geometries that require computational workflows with rapid and highly automated mesh generation. In order for the DIM class of methods to work correctly the spatial accuracy should be sufficient to resolve the interfaces correctly without an excessive diffusion across them and this requirement can be achieved by either increasing the spatial resolution at the interface regions or resorting to highresolution numerical methods. Additionally the numerical methods should be non-oscillatory since very strong gradients across material interfaces can result in spurious oscillations and a blown-up simulation. There are several high-order high-resolution numerical methods in the unstructured finite-volume framework such as the Weighted Essentially Non-Oscillatory (WENO) [17,63,75], Central Weighted Essentialy Non-Oscillatory (CWENO) [19,61], Multidimensional Optimal Order Detection (MOOD) [15,20], Monotone Upstream Scheme for Conservation laws (MUSCL) [52,57] and the Edge Based Reconstruction WENO (EBRWENO) [7].

In this study however we will be focusing on the application of the CWENO type of schemes as developed in $[19,61]$ since these methods were found to be more robust and significantly more computational efficient compared to the original WENO type of schemes for unstructured meshes, that have previously been successfully applied by Dumbser et al. [18] to the full seven equation Baer-Nunziato model. The improved robustness and efficiency is provided by the low-order polynomials associated with the directional stencils and their reduced size compared to the high-order polynomial associated with the central stencil. The reader is referred to $[19,61]$ for an overview of the methods. All the schemes/models are developed in the open source community UCNS3D solver [68], and we assess their performance in terms of for a series of stringent 2D and 3D test problems. The paper is organized as follows. In Sect. 2 we introduce the formulation of the governing equations 
employed for this study, followed by the numerical framework used to describe the highorder finite-volume framework for unstructured meshes, the reconstruction process for the CWENO schemes, while describing the chosen fluxes and temporal discretisation employed in Sect. 3. In Sect. 4 the numerical results obtained for all the test problems are presented and compared against analytical, reference or experimental solution whenever possible. Finally, the last section describes the conclusions drawn from this study.

\section{Governing Equations}

The quasi-conservative five-equation model of Allaire et al. [1] is considered in this study for inviscid compressible multicomponent flows. For two fluids this involves two continuity equations Eqs. (1) and (2), a momentum equation per dimension Eq. (3), an energy equation Eq. (4), and the non-conservative advection equation of the volume fraction of one of the two fluids Eq. (5) as given below:

$$
\begin{aligned}
& \frac{\partial\left(a_{1} \rho_{1}\right)}{\partial t}+\nabla \cdot\left(a_{1} \rho_{1} \mathbf{u}\right)=0, \\
& \frac{\partial\left(a_{2} \rho_{2}\right)}{\partial t}+\nabla \cdot\left(a_{2} \rho_{2} \mathbf{u}\right)=0, \\
& \frac{\partial \rho \mathbf{u}}{\partial t}+\nabla \cdot(\rho \mathbf{u u}+p \mathbf{I})=0, \\
& \frac{\partial E}{\partial t}+\nabla \cdot(E+p) \mathbf{u}=0, \\
& \frac{\partial a_{1}}{\partial t}+\mathbf{u} \cdot \nabla a_{1}=0,
\end{aligned}
$$

where $\rho$ is the density, $\mathbf{u}=(u, v, w)^{T}$ is the velocity, $p$ is the pressure, $E$ is the total energy and $a$ is the volume fraction. The widely use stiffened gas EOS is employed for closing the five-equation model. It has been primarily selected due to its application for flow problems involving gases, liquids and solids. The stiffened gas EOS characterises each fluid pressure as:

$$
p_{i}=\left(\gamma_{i}-1\right) \rho_{i} \epsilon_{i}-\gamma_{i} \pi_{\infty, i},
$$

where $\pi_{\infty, i} \geq 0$ is a reference pressure, and will be set to $\pi_{\infty}=0$ for gases. The total mass and $\rho \epsilon$ being given by the following equations:

$$
\begin{aligned}
& \rho=\sum_{i} a_{i} \rho_{i}, \\
& \rho \epsilon=\sum_{i} a_{i} \rho_{i} \epsilon_{i},
\end{aligned}
$$

where $\epsilon$ is the internal energy, with $\rho \epsilon=E-\frac{1}{2} \rho \mathbf{u u}$. The EOS of the mixture reads

$$
\begin{aligned}
& \xi=\frac{1}{\gamma-1}=\sum_{i} \frac{a_{i}}{\gamma_{i}-1}, \\
& \frac{\pi_{\infty} \gamma}{\gamma-1}=\sum_{i} a_{i} \frac{\pi_{\infty, i} \gamma_{i}}{\gamma_{i}-1}, \\
& p=(\gamma-1) \rho \epsilon-\gamma \pi_{\infty} .
\end{aligned}
$$


Finally, the non-conservative volume fraction advection Eq. (5) is rewritten in a mathematically equivalent form as introduced by Johnsen and Colonius [32]:

$$
\frac{\partial a_{1}}{\partial t}+\nabla \cdot\left(a_{1} \mathbf{u}\right)=a_{1} \nabla \cdot \mathbf{u}
$$

\section{Numerical Framework}

\subsection{Spatial Discretization}

Consider a 3D domain $\Omega$ consisting of conforming tetrahedral, hexahedral, prism, and pyramid cells each one of them indexed by a unique mono-index $i$, and the governing equations of the five-equation model written in vector form as follows:

$$
\frac{\partial}{\partial t} \int_{V_{i}} \mathbf{U} d V+\int_{\partial V_{i}} \mathbf{F}_{n} d S=\int_{V_{i}} \mathbf{s} d V
$$

where $\mathbf{U}=\mathbf{U}(\mathbf{x}, t)$ is the vector of conserved variables and the volume fraction of one species, $\mathbf{F}_{n}$ is the non-linear flux in the direction normal to the cells interface as given below:

$$
\mathbf{U}=\left[\begin{array}{c}
a_{1} \rho_{1} \\
a_{2} \rho_{2} \\
\rho u \\
\rho v \\
\rho w \\
E \\
a_{1}
\end{array}\right], \mathbf{F}_{n}=\left[\begin{array}{c}
a_{1} \rho_{1} u_{n} \\
a_{2} \rho_{2} u_{n} \\
\rho u u_{n}+n_{x} p \\
\rho v u_{n}+n_{y} p \\
\rho w u_{n}+n_{z} p \\
u_{n}(E+p) \\
a_{1} u_{n}
\end{array}\right],
$$

where $u_{n}$ is the velocity normal to the bounded surface area, defined by $u_{n}=n_{x} u+n_{y} v+n_{z} w$. The source term $\mathbf{s}$ is with regards to the term $a_{1} \nabla \cdot \mathbf{u}$ of Eq. (5). Following the approach of Johnsen and Colonius [32] the source term is numerically approximated as surface integral, rather than a volume one, while using the same velocity estimate as the one used for the evaluation of the fluxes as shown below:

$$
\int_{V_{i}} a_{1} \nabla \cdot \mathbf{u} d V \approx \int_{V_{i}} a_{1} d V \cdot \int_{\partial V_{i}}\left(u_{n}\right)^{\text {Riem. }} d S .
$$

Integrating Eq. (13) over the mesh element $i$ using a high-order explicit finite-volume formulation the following equation is obtained that incorporates the source term as previously defined:

$$
\frac{d \mathbf{U}_{i}}{d t}=\frac{1}{\left|V_{i}\right|} \sum_{j=1}^{N_{f}} \sum_{\alpha=1}^{N_{q p}}\left(F_{\mathbf{n}_{\mathbf{i j}}}\left(\mathbf{U}_{i j, L}^{n}\left(\mathbf{x}_{i j, \alpha}, t\right), \mathbf{U}_{i j, R}^{n}\left(\mathbf{x}_{i j, \alpha}, t\right)\right)-\mathbf{a}_{i, 1}^{n} \cdot u_{n}^{R i e m}\left(\mathbf{x}_{i j, \alpha}, t\right)\right) \omega_{\alpha}\left|S_{i j}\right|,
$$

where $\mathbf{U}_{i}$ is the volume averaged vector of variables

$$
\mathbf{U}_{i}=\frac{1}{\left|V_{i}\right|} \int_{V_{i}} \mathbf{U}(x, y, z) d V,
$$

and $F_{\mathbf{n}_{\mathbf{i j}}}$ is a numerical flux function in the direction normal to the cell interface between a considered cell $i$ and one of its neighbouring cells $j . N_{f}$ is the number of faces per element, 
$N_{q p}$ is the number of quadrature points used for approximating the surface integrals, $\left|S_{i j}\right|$ is the surface area of the corresponding face, and $\mathbf{U}_{i j, L}^{n}\left(\mathbf{x}_{i j, \alpha}, t\right)$ and $\mathbf{U}_{i j, R}^{n}\left(\mathbf{x}_{i j, \alpha}, t\right)$ are the high-order approximations of the solutions for cell $i$ and cell $j$ respectively. $\alpha$ corresponds to different Gaussian integration points $\mathbf{x}_{\alpha}$ and weights $\omega_{\alpha}$ over each face. $\mathbf{a}_{i, 1}^{n}$ corresponds to the volume averaged volume fraction of cell $i$ at time level $n$. The volume, surface and line integrals are numerically approximated by a suitable Gauss-Legendre quadrature.

\subsection{Reconstruction}

A high-order polynomial $p_{i}(x, y, z)$ of order $r$ can be built that provides $r+1$ order of accuracy for a cell $i$, by ensuring that it has the same average as a general quantity $\mathbf{U}_{i}$. This can be formulated as:

$$
\mathbf{U}_{i}=\frac{1}{\left|V_{i}\right|} \int_{V_{i}} p_{i}(x, y, z) d V .
$$

The present polynomial reconstruction is based upon the approaches of $[53,63]$, that have been applied to smooth and discontinuous flow problems $[2-5,20,46,49,50,57,59-62,64-$ 67], and only the key-components will be presented herein and the reader is referred to $[53,63]$ for further details. For the reconstruction, a transformation from physical space to a reference space as introduced by Dumbser et al. [16,17] is employed to reduce the scaling effects that occur at unstructured meshes consisting of elements of different shape and size. In particular we employ the decomposition strategy defined in $[63,64]$, where each element is decomposed into triangular (2D) or tetrahedral (3D) elements and using one of the decomposed elements as the reference element for transforming to the new system of coordinates. Let $\mathbf{v} \mathbf{x}_{i j}, j=1,2, \ldots J_{i}$ be the vertices of the considered 3D general element. Non tetrahedral elements are decomposed into tetrahedrals and one of them is chosen with $\mathbf{w}_{1}=\left(x_{1}, y_{1}, z_{1}\right), \mathbf{w}_{2}=\left(x_{2}, y_{2}, z_{2}\right), \mathbf{w}_{3}=\left(x_{3}, y_{3}, z_{3}\right)$ and $\mathbf{w}_{4}=\left(x_{4}, y_{4}, z_{4}\right)$ being its four vertices. The transformation from the Cartesian coordinates $x, y, z$ into a reference space $\xi, \eta, \zeta$ is given by the following equations:

$$
\left(\begin{array}{l}
x \\
y \\
z
\end{array}\right)=\left(\begin{array}{l}
x_{1} \\
y_{1} \\
z_{1}
\end{array}\right)+J \cdot\left(\begin{array}{l}
\xi \\
\eta \\
\zeta
\end{array}\right),
$$

with the Jacobian matrix given by:

$$
J=\left[\begin{array}{ccc}
x_{2}-x_{1} & x_{3}-x_{1} & x_{4}-x_{1} \\
y_{2}-y_{1} & y_{3}-y_{1} & y_{4}-y_{1} \\
z_{2}-z_{1} & z_{3}-z_{1} & z_{4}-z_{1}
\end{array}\right] .
$$

Using an inverse mapping the element $V_{i}$ can be transformed to the element $V_{i}^{\prime}$ in the reference co-ordinate system as:

$$
\mathbf{v} \mathbf{x}_{i j}^{\prime}=J^{-1} \cdot\left(\mathbf{v x}_{i j}-\mathbf{w}_{1}\right), \quad j=1,2, \ldots J_{i} .
$$

while the spatial average of the conserved variable $\mathbf{U}_{i}$ does not change during transformation

$$
\mathbf{U}_{i}=\frac{1}{\left|V_{i}\right|} \int_{V_{i}} \mathbf{U}(x, y, z) d V \equiv \frac{1}{\left|V_{i}^{\prime}\right|} \int_{V_{i}^{\prime}} \mathbf{U}(\xi, \eta, \zeta) d \xi d \eta d \zeta .
$$

The reconstruction polynomial uses a central stencil $\mathcal{S}^{1}$, which is built by recursively adding neighbouring elements, consisting of $M+1$ cells including the considered cell $i$. We 
employ the stencil based compact algorithm (SBC) introduced in [58], which is characteristed by a low computational cost and increased robustness in regions of poor quality meshing. In the present study we employ $M=2 K$, for enhanced robustness as reported in several previous studies $[15,30,58]$, where $K$ is the total number of polynomial coefficients given by:

$$
K(r, d)=\frac{1}{d !} \prod_{l=1}^{d}(r+l)
$$

where $d \in[2,3]$ is the space dimensions. The central stencil $\mathcal{S}^{1}$ is given by

$$
\mathcal{S}_{i}^{c}=\bigcup_{m=0}^{M_{c}} V_{m}
$$

where the index $m$ refers to the local numbering of the elements in the stencil with the element with index 0 being the considered cell $i$, and the index $c$ referring to the stencil number (in case of multiple stencils) where for the central stencil $c=1$. All the cells of each stencil are transformed in the reference space $\mathcal{S}_{i}^{\prime}$, where the $r$ th order reconstruction polynomial is an expansion over local polynomial basis functions $\phi_{k}(\xi, \eta, \zeta)$ given by:

$$
p(\xi, \eta, \zeta)=\sum_{k=0}^{K} a_{k} \phi_{k}(\xi, \eta, \zeta)=\mathbf{U}_{0}+\sum_{k=1}^{K} a_{k} \phi_{k}(\xi, \eta, \zeta)
$$

where $\mathbf{U}_{0}$ corresponds to the vector of conserved variables at the considered cell $i$, and $a_{k}$ are the degrees of freedom of the polynomial. The degrees of freedom $a_{k}$ for the polynomial for each cell $m$ are obtained by ensuring that the cell average of the reconstruction polynomial $p(\xi, \eta, \zeta)$ is equal to the cell average of the solution $\mathbf{U}_{m}$ :

$$
\int_{V_{m}^{\prime}} p(\xi, \eta, \zeta) d \xi d \eta d \zeta=\left|V_{m}^{\prime}\right| \mathbf{U}_{0}+\sum_{k=1}^{K} \int_{V_{m}^{\prime}} a_{k} \phi_{k} d \xi d \eta d \zeta=\left|V_{m}^{\prime}\right| \mathbf{U}_{m}, \quad m=1, \ldots, M
$$

The conservation condition in Eq. (18) is such that requires the basis functions to have a zero mean value over the considered transformed cell $V_{0}^{\prime}$. This can be achieved by using hierarchical orthogonal basis functions defined on a unit element in reference space. For triangular and tetrahedral elements hierarchical orthogonal basis functions can achieve this requirement. However for arbitrary shaped quadrilaterals, pyramids, prisms and hexahedrals they do not transform to a unit-element exactly, and therefore their basis functions need to be constructed in a way that the conservation condition in Eq. (18) is satisfied. The basis functions employed $\phi_{k}$ for all the elements in the stencil are defined as follows and satisfy this requirement:

$$
\phi_{k}(\xi, \eta, \zeta) \equiv \psi_{k}(\xi, \eta, \zeta)-\frac{1}{\left|V_{0}^{\prime}\right|} \int_{V_{0}^{\prime}} \psi_{k} d \xi d \eta d \zeta \quad k=1,2, \ldots, K,
$$

and in the present study $\psi_{k}$ are Legendre polynomials basis functions. Denoting the integrals of the basis function $k$ over the cell $m$ in the stencil, and the vector of right-hand side by $A_{m k}$ and $b$ respectively as given by 


$$
A_{m k}=\int_{V_{m}^{\prime}} \phi_{k} d \xi d \eta d \zeta, \quad b_{m}=\left|V_{m}^{\prime}\right|\left(\mathbf{U}_{m}-\mathbf{U}_{0}\right),
$$

the equations for degrees of freedom $a_{k}$ can be rewritten in a matrix form as:

$$
\sum_{k=1}^{K} A_{m k} a_{k}=b_{m}, \quad m=1,2, \ldots M .
$$

The resulting linear system is solved by a QR decomposition based on Householder transformation while using a Moore-Penrose pseudo-inverse of $A_{m k}$ which is only computed once at the beginning of the simulation as detailed in [58].

\subsubsection{CWENO Scheme}

The CWENO scheme employed follows the implementation of Tsoutsanis and Dumbser [61] and is the combination of an optimal (high-order) polynomial $p_{o p t}$ with lower-order polynomials. The reconstruction for the optimal (high-order) polynomial uses an expanded central stencil, (suitable for the desired polynomial order) while the reconstruction for the lower-order polynomials employs the compact directional stencils. When the variation of the solution is smooth across all the stencils, the optimal polynomial is recovered and therefore the desired-order of accuracy is obtained. At the presence of discontinuous data at least one of the directional stencils associated with the lower-order polynomials could contain smooth data, hence it is going to have the largest influence in the reconstruction. All the polynomials involved satisfy the requirement of matching the cell averages of the solution, therefore they are solved with the same constrained least-squares technique. The directional stencils employ the Type 3 definition which includes one directional stencil per element face as detailed in the work by Tsoutsanis [58]. The optimal polynomial is defined as follows:

$$
p_{o p t}(\xi, \eta, \zeta)=\sum_{s=1}^{s_{t}} \lambda_{s} p_{s}(\xi, \eta, \zeta)
$$

where $s$ is the stencil index, with $s=1$ being the central, $s=2,3$, being the directional, $s_{t}$ being the total number of stencils, and $\lambda_{s}$ being the linear coefficients for each stencil, whose sum is equal to 1 . The $p_{1}$ polynomial is not computed directly, but computed by subtracting the lower-order polynomials from the optimum polynomial as follows:

$$
p_{1}(\xi, \eta, \zeta)=\frac{1}{\lambda_{1}}\left(p_{o p t}(\xi, \eta, \zeta)-\sum_{s=2}^{s_{t}} \lambda_{s} p_{s}(\xi, \eta, \zeta)\right) .
$$

The CWENO reconstruction polynomial is given as a non-linear combination of all the polynomials in the following manner:

$$
p(\xi, \eta, \zeta)^{\mathrm{cweno}}=\sum_{s=1}^{s_{t}} \omega_{s} p_{s}(\xi, \eta, \zeta),
$$

where $\omega_{s}$ correspond to the non-linear weights assigned to each polynomial, and in regions with smooth data $\omega_{s} \approx \lambda_{s}$, hence obtaining the high-order approximation from the central stencil, and in regions of discontinuous solutions the reconstructed solution will be mostly 
influenced from the lower-order polynomials of the directional stencils. where $\tilde{a}_{k}$ are the reconstructed degrees of freedom; and the non-linear weight $\omega_{s}$ is defined as:

$$
\omega_{s}=\frac{\tilde{\omega}_{s}}{\sum_{s=1}^{s_{t}} \tilde{\omega}_{s}} \quad \text { where } \quad \tilde{\omega}_{s}=\frac{\lambda_{m}}{\left(\epsilon+\mathcal{S} \mathcal{I}_{s}\right)^{b}} .
$$

The smoothness indicator $\mathcal{S} \mathcal{I}_{m}$ is given by:

$$
\mathcal{S} \mathcal{I}_{s}=\sum_{1 \leq|\beta| \leq r} \int_{V_{0}^{\prime}}\left(\mathcal{D}^{\beta} p_{s}(\xi, \eta, \zeta)\right)^{2}(d \xi, d \eta, d \zeta)
$$

where $\beta$ is a multi-index, $r$ is the polynomial's order, $\lambda_{m}$ is the linear weight. The value set to prevent division by zero of $\epsilon=10^{-6}$ is used, with $b=4$ and $\mathcal{D}$ being the derivative operator. The smoothness indicator is a quadratic function of the degrees of freedom $\left(a_{k}^{s}\right)$ and Eq. (33) can be rewritten as:

$$
\mathcal{S I}_{s}=\sum_{k=1}^{K} a_{k}^{s}\left(\sum_{q=1}^{K} \mathcal{O I}_{k q} a_{q}^{s}\right)
$$

where the oscillation indication matrix $\mathcal{O} \mathcal{I}_{k q}$ is given by:

$$
\mathcal{O} \mathcal{I}_{k q}=\sum_{1 \leq|\beta| \leq r} \int_{V_{0}^{\prime}}\left(\mathcal{D}^{\beta} \phi_{k}(\xi, \eta, \zeta)\right)\left(\mathcal{D}^{\beta} \phi_{q}(\xi, \eta, \zeta)\right)(d \xi, d \eta, d \zeta)
$$

which can be precomputed and stored at the beginning of the simulation. For the directional stencils and their corresponding polynomials we employ $r=1$ to obtain 2 nd-order of accuracy, and any arbitrary order of accuracy for the polynomial associated with the central stencil. The linear weights are computed by firstly assigning the non-normalised linear weight for the central stencil $\lambda_{1}^{\prime}$ an arbitrary value, and then normalising this as follows:

$$
\lambda_{1}=1-\frac{1}{\lambda_{1}^{\prime}},
$$

with the linear weights associated with lower-order polynomials being equal and provided by the following expression:

$$
\lambda_{s}=\frac{1-\lambda_{1}}{s_{t}-1}
$$

where $s_{t}$ is the total number of stencils. From this point forward the order of the scheme will be defined by a number next to the type of the scheme, such as CWENO3 and WENO4 corresponding to a 3rd-order CWENO scheme and a 4th-order WENO scheme respectively.

\subsection{Fluxes Approximation and Temporal Discretisation}

For the inviscid fluxes the approximate Harten-Lax-van Leer-Contact (HLLC) Riemann solver of Toro [54] is employed, unless otherwise stated. The temporal discretisation employs the 3rd-order explicit Strong Stability Preserving (SSP) Runge-Kutta method [26] which is stable for $C F L \leq 1$. All the volume/surface/line integrals are approximated by Gaussian quadrature rule suitable for the order of polynomial employed. It has to be noted that the reconstruction in the present study is carried out with respect to the conserved variables or 
primitive variables by transforming to either one of them only during the reconstruction process, while always transforming to conservative variables prior to the fluxes calculation. All the schemes developed are implemented in the open-source UCNS3D CFD code [68] using object-oriented Fortran 2003, and employing MPI message passing interface (MPI), and the Open Multi-Processing (OpenMP) application programming interface (API). The reader is referred to $[56,67]$ for more details on implementation and performance benchmarks.

\section{Applications}

We present the numerical simulations employed to assess the performance of the CWENO interface capturing schemes for the solution of the inviscid compressible Euler equations for multicomponent flows. Several benchmark test problems have been performed.

\subsection{Multi-Species Convergence Test}

For verifying the designed order of spatial accuracy for the numerical schemes developed, a multi-species advection test similar to the one employed by Wong and Lele [73] is used. In this test a smooth volume fraction initial profile of two gases is advected for one period in a periodic computational domain. The initial condition is given by:

$$
\left(\rho_{1}, \rho_{2}, u, v, p, a_{1}\right)=(7,1,1,0,1 / 1.4,0.5+0.25 \sin (2 \pi(x-0.5))) .
$$

The $2 \mathrm{D}$ computational domain $[0,1]^{2}$ consists of arbitrary unstructured triangular elements of 10, 20, 40 and 80 edges per side resolution as shown in Fig. 1, and the simulation is run for a time of $t_{f}=1$. The two gases selected are nitrogen and helium with specific heats 1.4 and 1.66 respectively. The numerical errors $e_{L^{2}}$ and the $e_{L^{\infty}}$ are computed as follows:

$$
\begin{aligned}
e_{L^{2}} & =\sqrt{\frac{\sum_{i} \int_{\Omega_{i}}\left(\mathbf{U}_{e}\left(x, t_{f}\right)-\mathbf{U}_{c}\left(x, t_{f}\right)\right)^{2} d V}{\sum_{i}\left|\Omega_{i}\right|}}, \\
e_{L^{\infty}} & =\mathcal{M a x} \mid\left(\mathbf{U}_{e}\left(x, t_{f}\right)-\mathbf{U}_{c}\left(x, t_{f}\right) \mid,\right.
\end{aligned}
$$

where $\mathbf{U}_{c}\left(x, t_{f}\right)$ and $\mathbf{U}_{e}\left(x, t_{f}\right)$ are the computed and exact solutions at the end of the simulation $t_{f}=1.0$. The exact solution $\mathbf{U}_{e}\left(x, t_{f}\right)$ being given by the initial condition itself at $t=0$. The simulations were performed using $C F L=0.1$ in order to ensure a sufficiently small time-step size so that the schemes are not restricted by the time-discretisation for achieving the designed order of spatial accuracy. The wallclock time per simulation is normalised with respect to the fastest time taken for a simulation, which in this setup is provided by the CWENO3 scheme on the coarsest mesh.

From the obtained convergence orders of the schemes as shown in Table 1 it is clear that the schemes achieve their designed order of convergence for both $e_{L^{\infty}}$ and $e_{L^{2}}$. The desirable feature of the CWENO variant is that it comes with significant lower computational costdue to the reduction of the size of the directional stencils - compared to the WENO schemes. For this test the CWENO schemes require approximately $60-80 \%$ of the time taken by a WENO scheme of the same spatial order, a figure which is expected to improve when larger mesh sizes, and 3D test problems are deployed. 


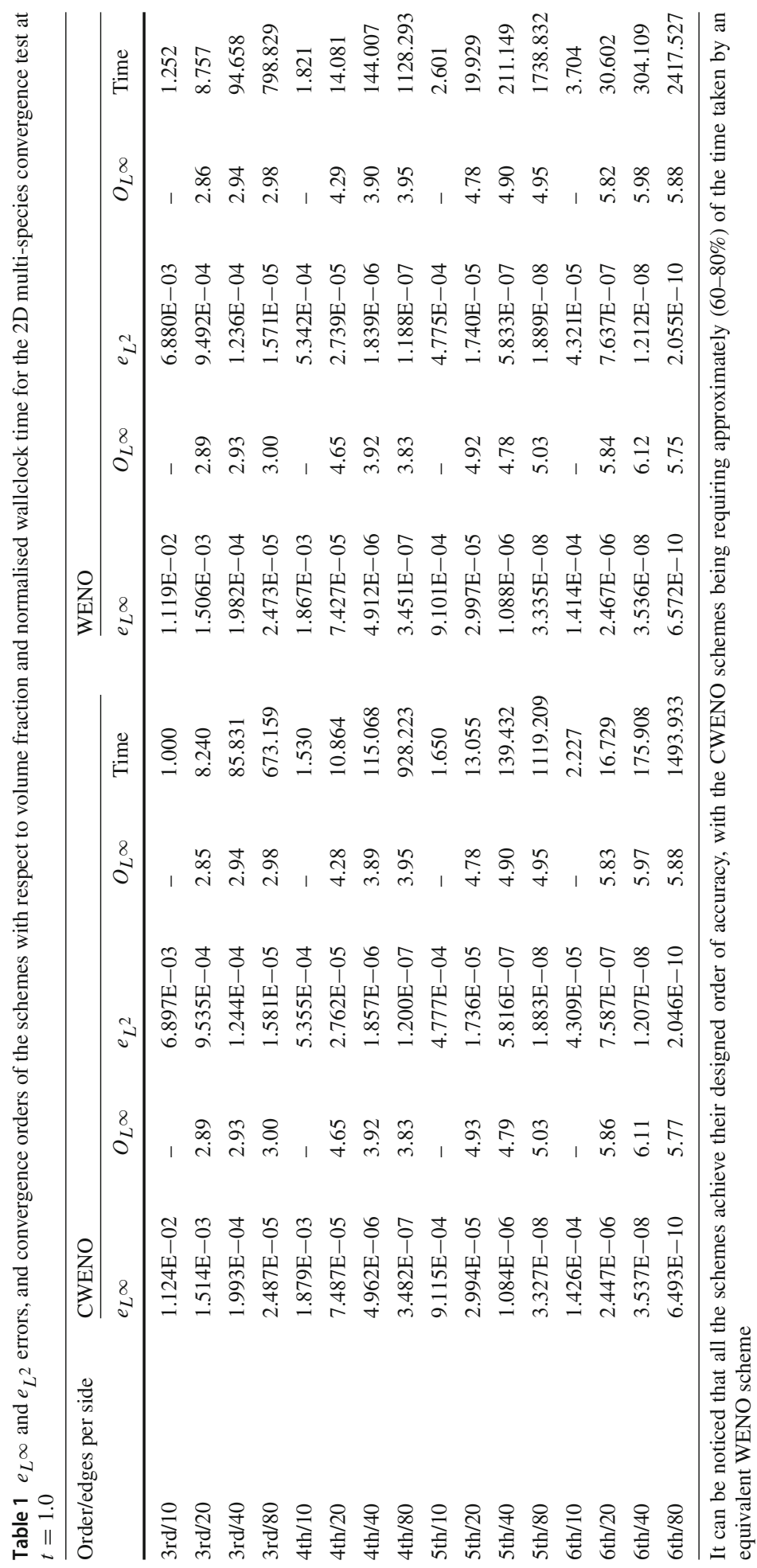



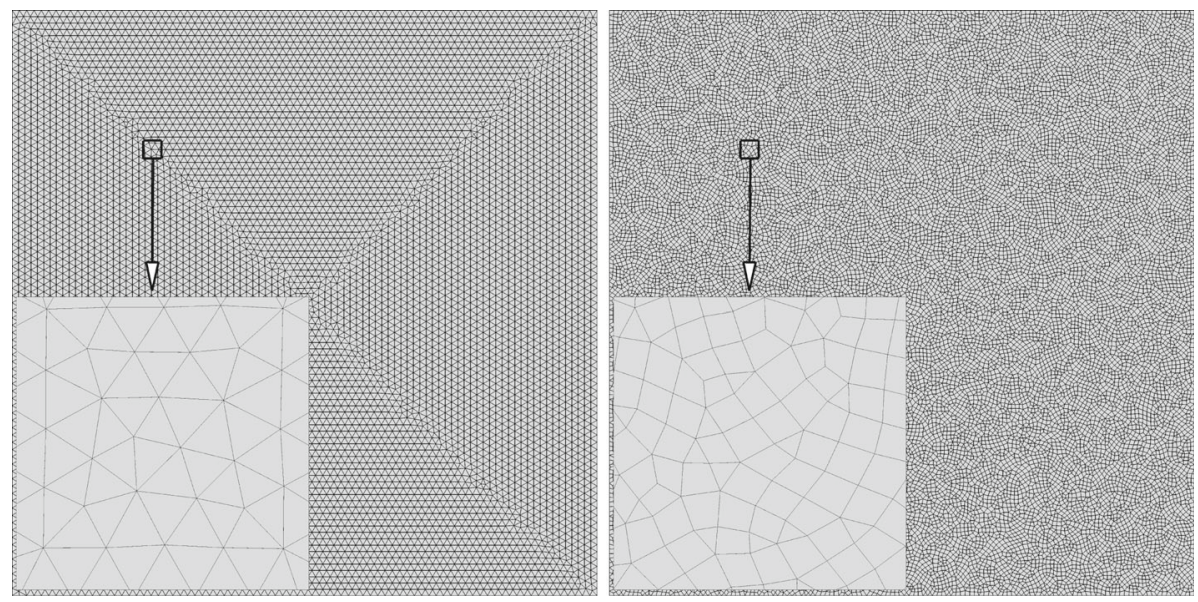

Fig. 1 Typical examples of 2D unstructured meshes used, where a zoomed region provides details of the structure for a triangular mesh (left) and a mixed-element mesh (right)

\subsection{Isolated Material Interface}

For assessing the non-oscillatory properties of the considered schemes, the advection of a sharp material interface within a periodic domain is considered. A sharp material interface is frequently encountered in several multicomponent flows, hence the robustness of the proposed methods is of paramount importance, for the successful deployment of these schemes in multicomponent flows. The material interface is advected with constant velocity across the computational domain, and the pressure and temperature is also constant across the interface. The initial conditions are given by:

$$
\left(\rho, u, v, p, \gamma, a_{1}\right)= \begin{cases}(10.0,0.5,0,1 / 1.4,1.4,1), & \text { if } x<0.5 \\ (1.0,0.5,0,1 / 1.4,1.66,0), & \text { otherwise }\end{cases}
$$

The 2D computational domain $[0,1]^{2}$ consists of a mixed-element mesh as shown in Fig. 1 consisting of triangular and quadrilateral mesh elements of 80 edges per side resolution, and the simulation is run for a time of $t_{f}=2$. Firstly a comparison between 3rd-order CWENO3 and WENO3 is made to assess the non-oscillatory properties of the schemes and as it can be noticed from the obtained results in Fig. 2 the CWENO3 is significantly less oscillatory near the material interface. The relative coarse resolution of the present mesh, is intentionally selected to highlight one of the key differences between the two schemes, which is the compactness of their directional stencils. In the CWENO the directional stencils correspond to lower-order polynomials compared to WENO schemes and therefore are significantly more compact, therefore resulting in improved robustness in such situations. Secondly the influence of two types of reconstructions are explored with respect to the conserved and primitive variables. As it can be seen in Fig. 3, the solutions obtained with the primitive variable reconstruction are free from any oscillations in the pressure and velocity solution. It is well documented [31-34] that primitive variable reconstruction is needed to prevent spurious oscillations in problems where $\gamma$ is not constant, since reconstruction in conservative or characteristic variables suffer from oscillations across them. Finally the CWENO scheme with primitive variables employed provide the correct solution with no oscillations, and as seen in 

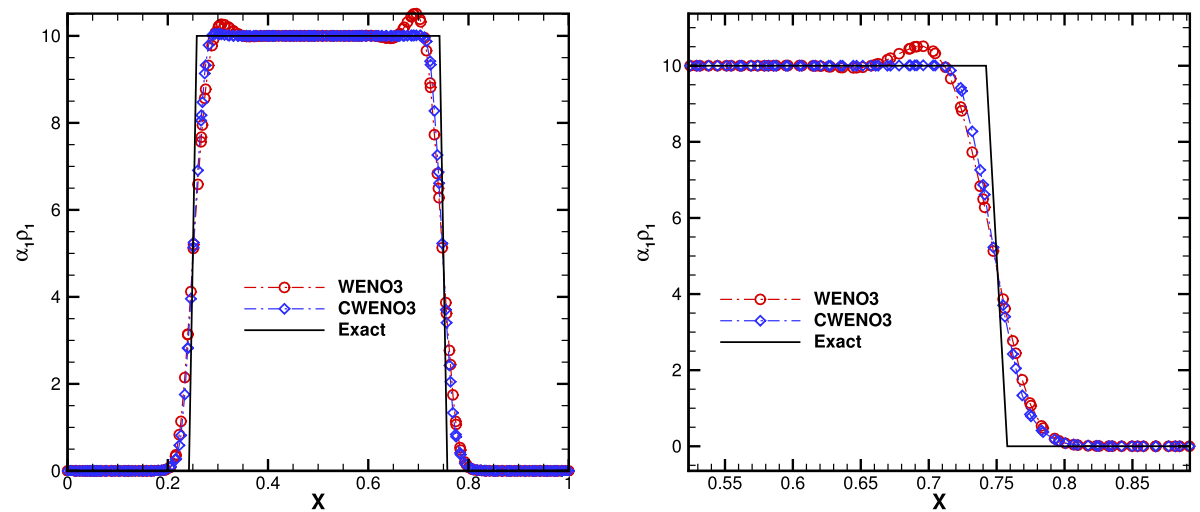

Fig. 2 Plots of density $a_{1} \rho_{1}$ for the isolated sharp-material interface at $t=2$ obtained with WENO3 and CWENO3 schemes, and compared with the reference exact solution. It can be noticed that the the WENO3 is producing some oscillations near the material interface, while they are absent from the CWENO3 obtained solution
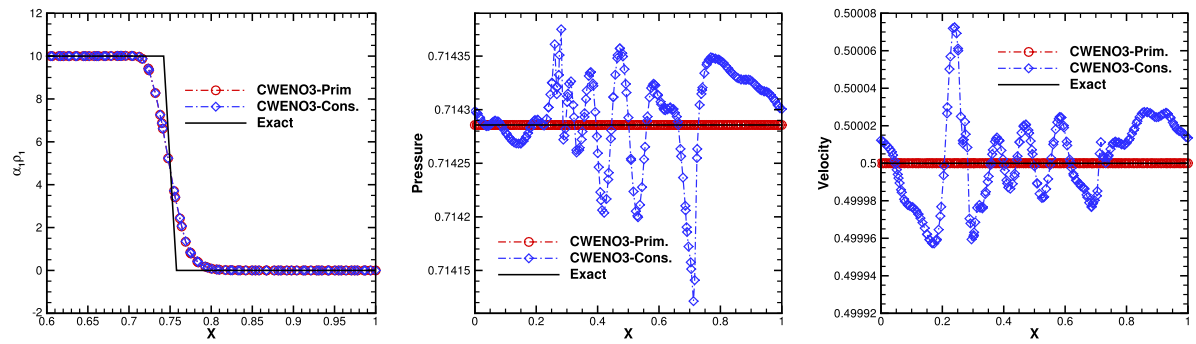

Fig. 3 Plots of density $a_{1} \rho_{1}$, pressure and velocity for the isolated sharp-material interface at $t=2$ obtained with the CWENO3 scheme with primitive and conservative variables reconstruction, and comparison with the exact solution. The primitive variable reconstruction is producing an oscillation-free solution for both pressure and velocity
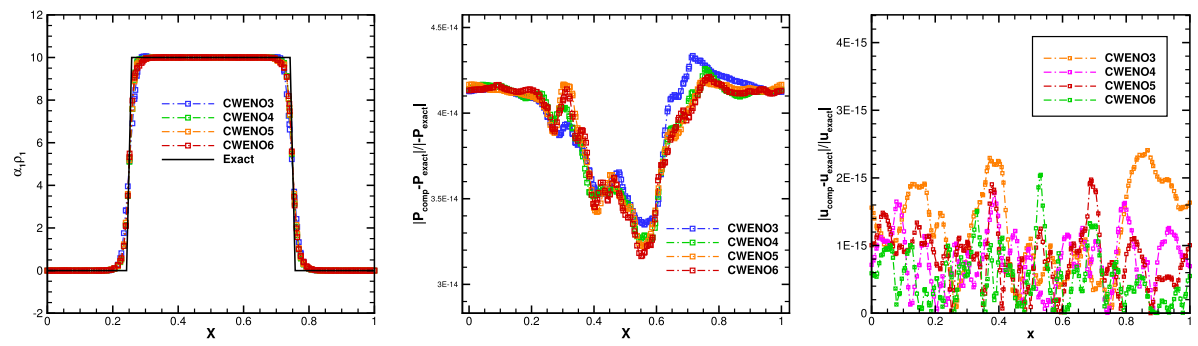

Fig. 4 Plot of density $a_{1} \rho_{1}$, pressure and velocity for the isolated sharp-material interface at $t=2$ obtained with several CWENO schemes where it can be noticed that all the schemes provide the correct solution with no oscillations, and normalised pressure and velocity error at the $t=2$ where it can be seen that the minute oscillations are close to machine precision

Fig. 4 the minute oscillations in normalised pressure and velocity errors are close to machine precision. Therefore unless otherwise stated from this point onwards the reconstruction with respect to primitive variables is going to be employed. 


\subsection{Gas-Liquid Riemann Problem}

The 2D equivalent of the well established gas liquid Riemann problem of Cocchi et al. [12] is deployed in this study. It is an ideal test problem for exposing the performance of the developed numerical schemes in for stiffened gas EOS. The left state of the problem consists of highly compressed air, and the right state is water at atmospheric pressure. The initial conditions for this problem following the non-dimensionalisation of Cocchi et al. [12] are:

$$
\left(\rho_{1}, \rho_{2}, u, v, p, a_{1}\right)= \begin{cases}(1.241,0,0,2.753,1.4,1), & \text { if } \mathbf{x}<0 \\ \left(0,0.991,0,3.059 \times 10^{-4}, 0\right), & \text { otherwise } .\end{cases}
$$

The specific heats for air and water are 1.4 and 5.5 respectively, with the $\pi_{\infty}=1.505$ for water. The $2 \mathrm{D}$ computational domain $[-1,1]^{2}$ is discretised by a mixed-element unstructured mesh similar to the one shown in Fig. 1 which consists of arbitrary triangular and quadrilateral elements of 100 edges per side resolution, and the simulation is run for a time of $t_{f}=$ 0.2. Two schemes are employed for this test problem a WENO3 and a CWENO5. The CWENO5 scheme employs a higher-order polynomial for the central stencil compared to the WENO3 order scheme, and a lower-order polynomial (and stencil size) than the WENO3 order scheme. As it can be noticed from the obtained results shown in Fig. 5 while both schemes provide results in good agreement with the exact solution, the CWENO5 order scheme is less oscillatory in this coarse grid resolution due to the compactness offered by the smaller directional stencils. Therefore from this point onwards only the CWENO schemes will be employed in the following test problems.

\subsection{Gas Bubble in Water}

A problem that has been frequently used $[25,39]$ to assess the performance of various numerical methods is considered. This problem involves very high-pressure due to the collapse of a gas bubble in a liquid. These types of problems are typically found in applications such as fuel injectors, naval propulsion systems and shockwave lithotripsy. In this study a strong shock $M_{s h}=1.72$ is travelling through water and moving towards an air bubble. The initial condition is given by:

$$
\left(\rho, u, v, p, \gamma, \pi_{\infty}, a_{1}\right)= \begin{cases}\left(1323.65,681.058,0,1.9 \cdot 10^{9}, 4.4,6 \cdot 10^{8}, 0\right), & \text { for Post-shock } \\ \left(1000,0,0,10^{5}, 4.4,6 \cdot 10^{8}, 0\right), & \text { for Pre-shock } \\ \left(1,0,0,10^{5}, 1.4,0,1\right), & \text { for Bubble }\end{cases}
$$

The domain is a $[0,0.024]^{2}$ square, the gas bubble is placed at the centre of the domain $(0.012 \mathrm{~m}, 0.012 \mathrm{~m})$, and the interface between shocked and unshocked regions placed at $x=0.0066 \mathrm{~m}$. Non-reflecting boundary conditions are prescribed at the left and right boundaries of the domain, while an Euler slip-wall is prescribed for the top and bottom boundaries. The domain is discretised by an quadrilateral mesh with approximately 1.0 million cells and an average edge length of $e_{c} \approx D_{b} / 250$, where $D_{b}$ is the diameter of the gas bubble $D_{b}=6 \mathrm{~mm}$. The CWENO5 scheme was employed for this test problem, and the simulation is run until $t=6.2 \mu \mathrm{s}$.

The evolution of the bubble dynamics obtained with the present schemes is illustrated in Fig. 6 and in Fig. 7 where all the expected features including the reflected rarefaction wave, the water jet, the blast wave and the secondary jets are well captured and are similar to 

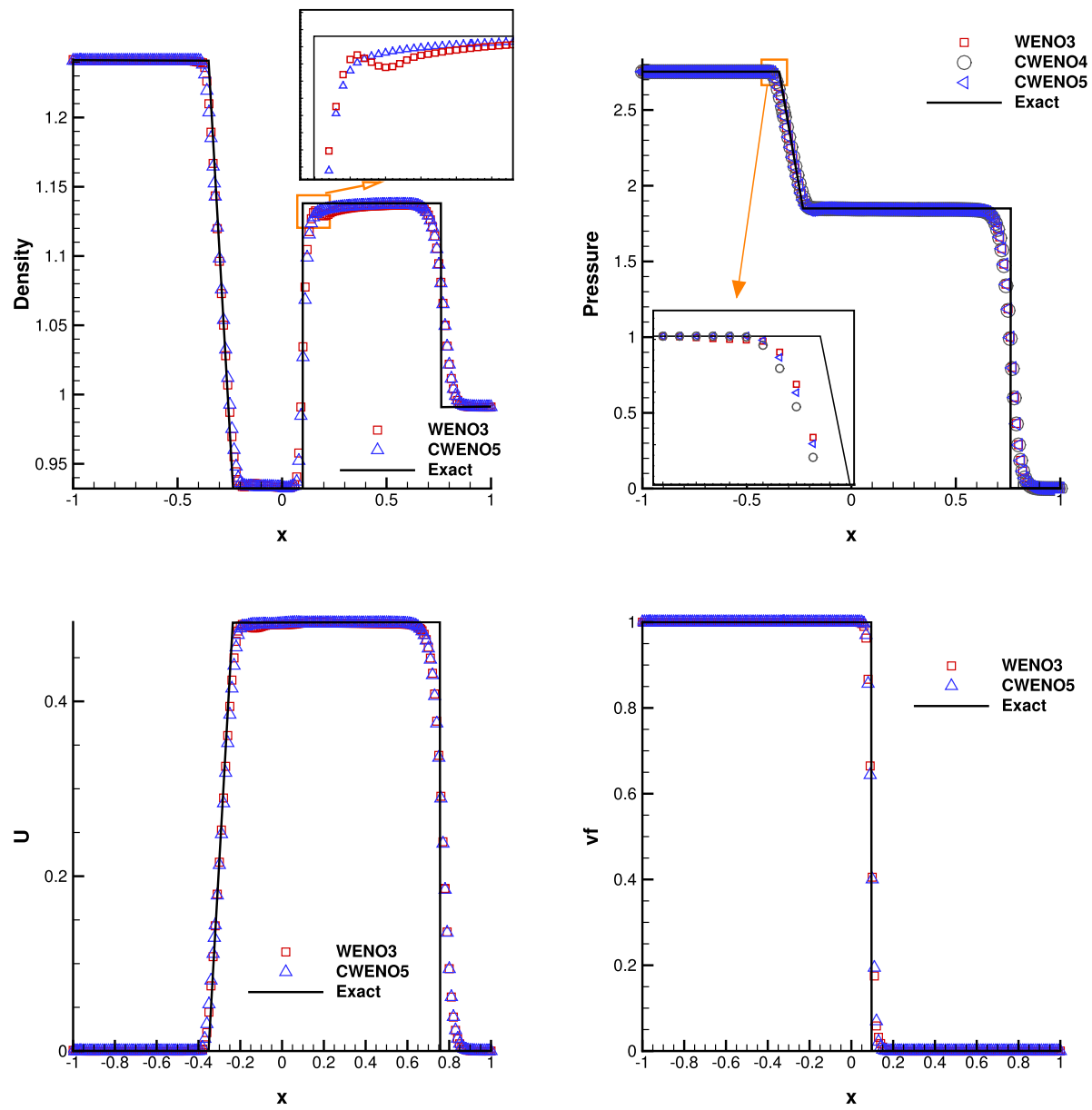

Fig. 5 Plots of density, pressure, velocity and volume fraction for the gas-liquid Riemann problem at $t=0.2$ obtained with WENO3, and CWENO5 schemes and compared with the exact solution. It can be noticed that the CWENO5 order scheme is less prone to oscillations compared to the WENO3 order scheme

the ones obtained by others Nourgaliev et al. [39] and Goncalves et al. [25]. The maximum pressure obtained in the computational domain is 7.7 $\mathrm{Fa}$, which is inline with the findings of other studies where higher pressures are seen in fine grid resolutions with Nourgaliev et al. [39] reporting 10.1GPa at a grid resolution of $e_{c} \approx D_{b} / 800$, and lower peak pressures at lower grid resolutions with Goncalves et al. [25] reporting a peak pressure of 7.8G $P a$ at a grid resolution of $e_{c} \approx D_{b} / 300$. Similarly the peak temperature at the time of lowest volume obtained is $\approx 15,670 \mathrm{~K}$ which is within the range obtained from the other studies [25,39].

The time evolution of the pressure along the centreline of the computational domain can be seen in Fig. 8, where it can be noticed that the first peak is associated with the blast wave at $t \approx 3.9 \mu$ s and the second peak a later time $t \approx 4.9 \mu$ s occurs when the blast wave collides with the bubble fragments as also reported by Goncalves et al. [25]. Finally the time history of the non-dimensional volume of the gas bubble is illustrated in Fig. 8, where it can be noticed that the results obtained are in good agreement with the results of Nourgaliev et al. 


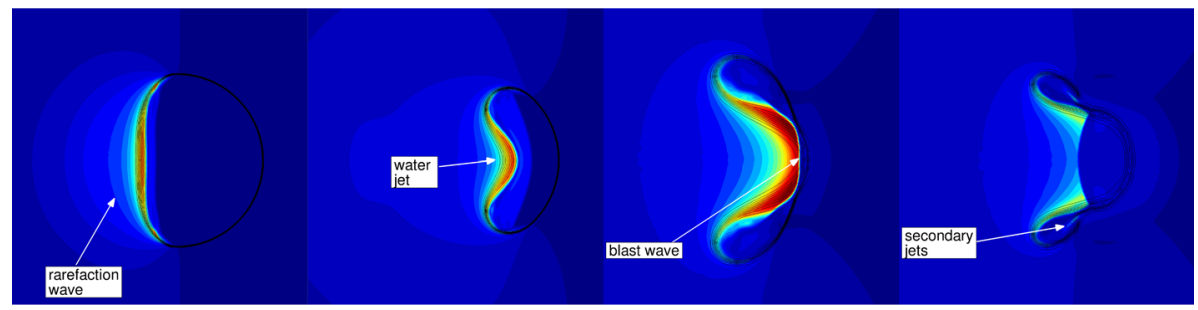
(a) $t=1.9 \mu \mathrm{s}$
(b) $t=3.1 \mu \mathrm{s}$
(c) $t=3.8 \mu \mathrm{s}$
(d) $t=4.1 \mu \mathrm{s}$

Fig. 6 Plots of the Mach number (contours) and volume fraction (lines) for the gas bubble collapse test problem at different instants. It can be noticed that all the expected features are captured using the present CWENO5 scheme

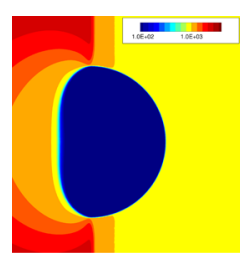

(a) $t=1.9 \mu \mathrm{s}$

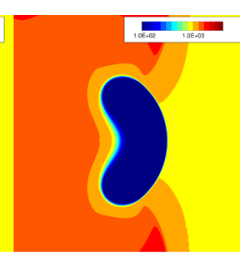

(b) $t=3.0 \mu \mathrm{s}$

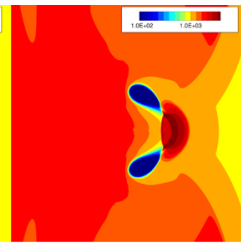

(c) $t=4.0 \mu \mathrm{s}$

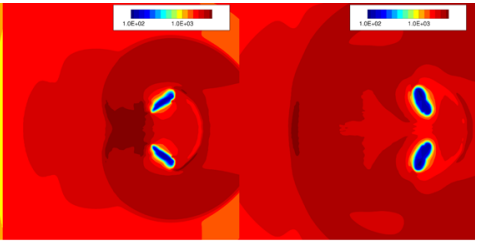

(d) $t=5.0 \mu \mathrm{s}$

(e) $t=5.7 \mu \mathrm{s}$

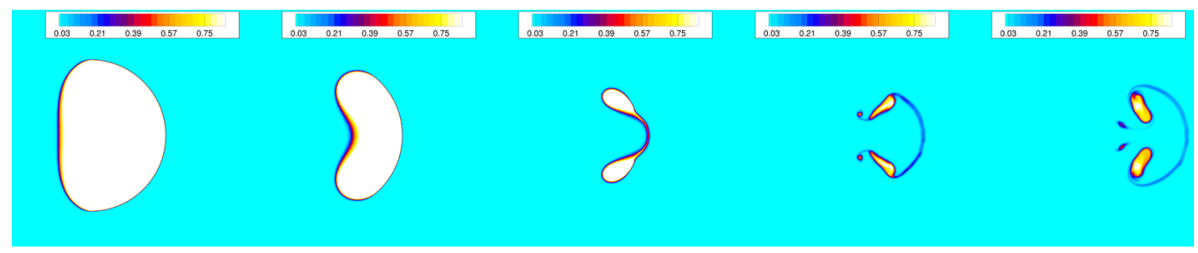
(f) $t=1.9 \mu \mathrm{s}$
(g) $t=3.0 \mu \mathrm{s}$
(h) $t=4.0 \mu \mathrm{s}$
(i) $t=5.0 \mu \mathrm{s}$
(j) $t=5.7 \mu \mathrm{s}$

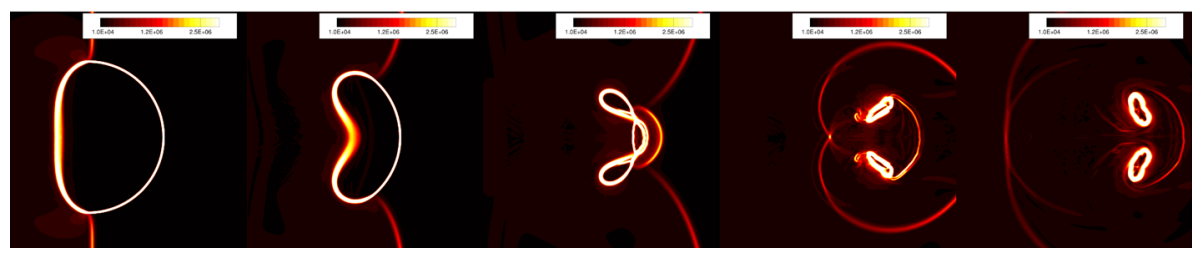
(k) $t=1.9 \mu \mathrm{s}$
(l) $t=3.0 \mu \mathrm{s}$
(m) $t=4.0 \mu \mathrm{s}$
(n) $t=5.0 \mu \mathrm{s}$
(o) $t=5.7 \mu \mathrm{s}$

Fig. 7 Plots of the density contours (top), volume fraction (middle), and density gradient magnitude (bottom) for the gas bubble collapse test problem at different instants

[39] capturing the correct compression slope, and rebound at late times. However due to the coarser grid-resolution employed in this study the maximum compression of the bubble is lower compared to the one obtained by Nourgaliev et al. [39].

\subsection{Water Column in Air}

The shock wave interaction with a cylindrical water column test case of Xiang and Wang [74] is employed in this study, and in essence it is the opposite of the gas-bubble collapse in water 

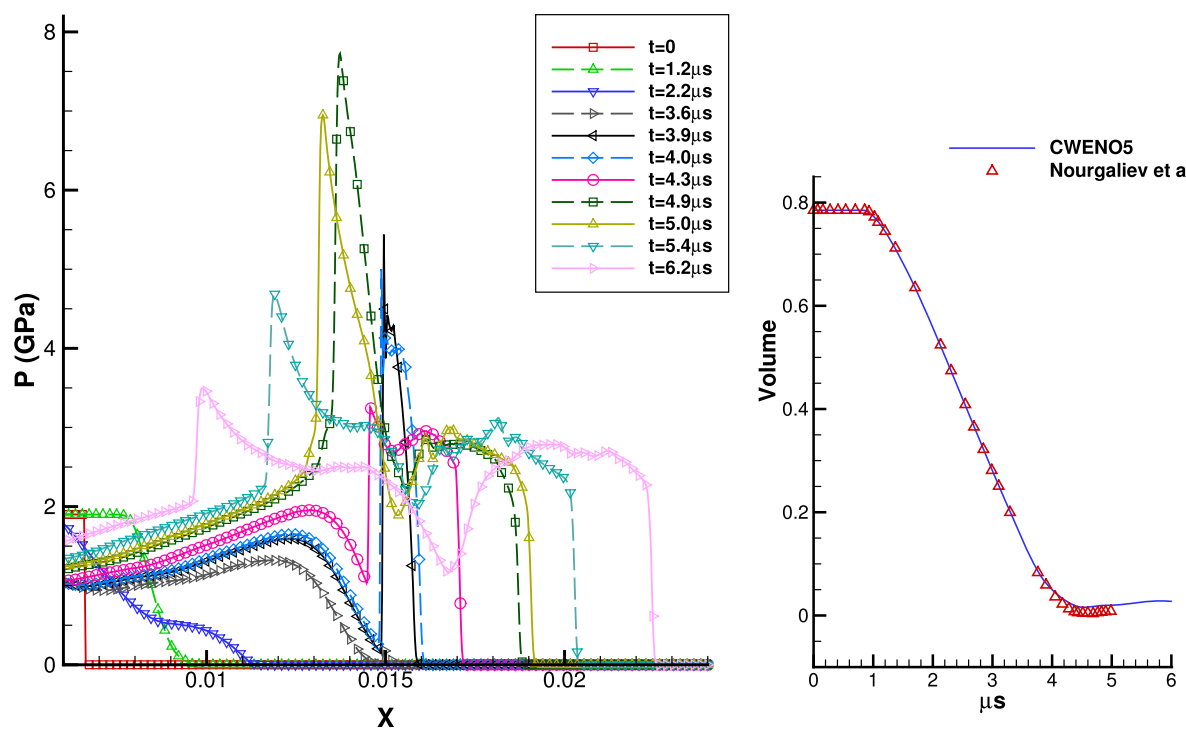

Fig. 8 Plots of pressure evolution along the centreline of the computational domain (left), and evolution of the non-dimensional volume of the gas bubble (right) for the gas bubble collapse in water test problem

problem seen previously. In this test a water droplet is surrounded by air, where a shockwave at $M_{s h}=2.4$ is moving towards the water bubble. The subject test serves as an ideal computational test-problem for development and assessment of numerical methods for interface dynamics of compressible multi-fluid flows with several practical applications including the liquid jet atomization in a scramjet engines, combustion, and supernova explosion. We are considering two variants of the water column in this test one without an air cavity, and one with an air cavity as performed by Xiang and Wang [74]. The setup of the test problem can be seen in Fig. 9, where for the water column without the air cavity $r=0$, while with air cavity $r=3.6 \mathrm{~mm}$.

The initial condition is given by:

$$
\left(\rho, u, v, p, \gamma, \pi_{\infty}, a_{1}\right)= \begin{cases}\left(3.85,567.3,0,0.664 \cdot 10^{6}, 1.4,0,0\right), & \text { for Post-shock } \\ \left(1.2,0,0,0.101 \cdot 10^{6}, 1.4,0,0\right), & \text { for Pre-shock } \\ \left(1000,0,0,10^{5}, 6.12,0.343 \cdot 10^{9}, 1\right), & \text { for Water }\end{cases}
$$

The domain is $x \in[0,0.2208], y \in[0,0.1152]$, the water column is centred at $(0.0576$ $\mathrm{m}, 0.0576 \mathrm{~m})$, and the interface between shocked and unshocked regions placed at $x=0.05$ $\mathrm{m}$. Non-reflecting boundary conditions are prescribed at the left and right boundaries of the domain, while an Euler slip-wall is prescribed for the top and bottom boundaries. The domain is discretised by an quadrilateral mesh with approximately 0.89 million cells and an average edge length of $e_{c} \approx D_{b} / 192$, where $D_{b}$ is the diameter of the water column/or the outer diameter when a cavity is included $D_{b}=96 \mathrm{~mm}$. The CWENO5 scheme was employed for this test problem, and the simulation is run until the non dimensional $t^{*}=t u / D_{b}=10$ where $u$ corresponds to the initial velocity behind the shock. The obtained computed density gradient magnitude for both variants are in good agreement with the numerical results of Xiang and Wang [74] as shown in Fig. 10. All the key flow features (reflected expansion wave, the transmitted wave, the Mach stem, and re-circulation zone) are correctly captured 

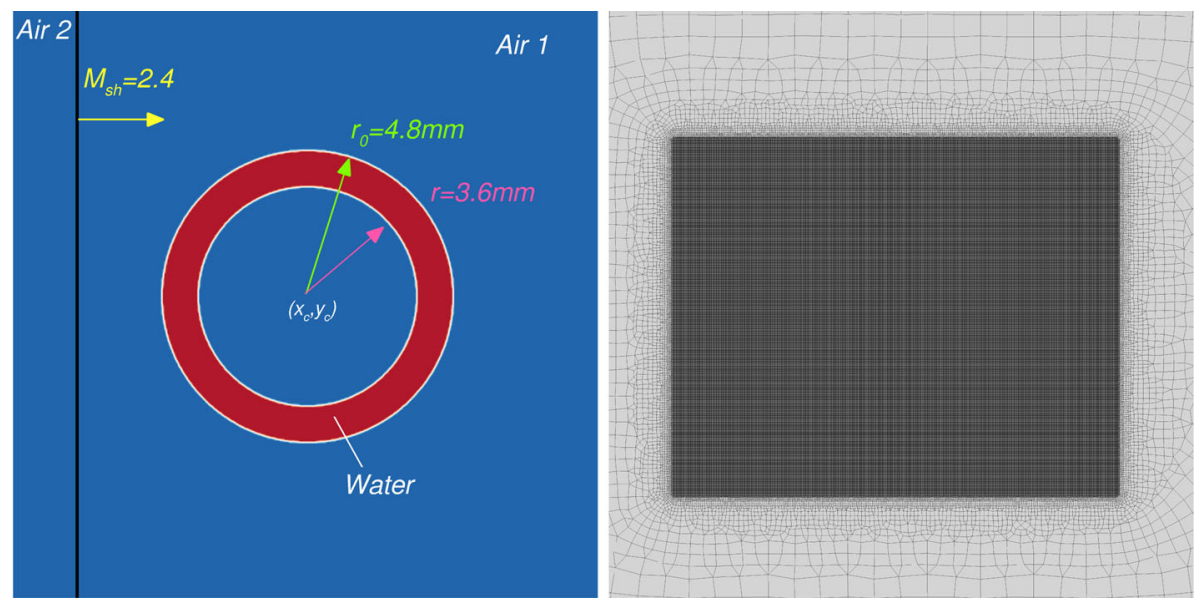

Fig. 9 Schematic diagram of the setup for the shock wave cylindrical water column test problem (left), and the corresponding mesh refinement used for the interaction zone (right)
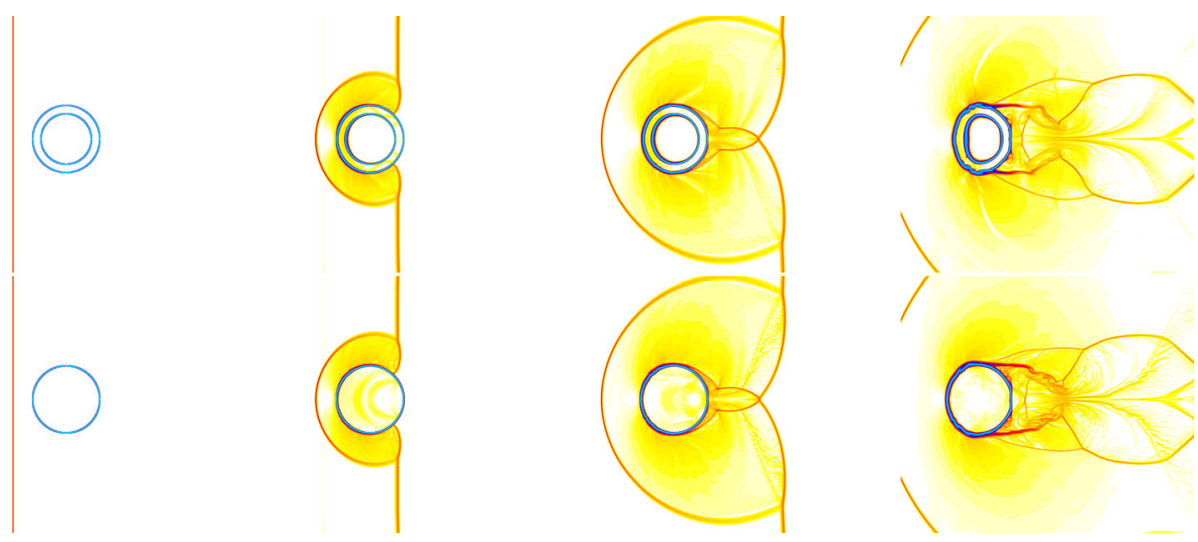

Fig. 10 Contour plots of density gradient magnitude of the computed solution of the shock wave interaction with a water column with a cavity (top) and without a cavity (bottom), at various instants $t^{*}=0, t^{*}=0.8$, $t^{*}=1.62, t^{*}=3.02$ (left to right). It can be noticed that all the expected flow features are captured by the present CWENO scheme

as shown in comparison with the experimental results for the water column without cavity of Sembian et al. [48] in Fig. 11.

As expected at late times the flow separates for both variants as seen in Fig. 12, where the water column without the cavity is compressed continuously while it gets flattened downstream as documented by Xiang and Wang [74] and Meng and Colonius [37]. For the water column with cavity the transverse jet formation and the associated Richtmyer-Meshkov instability occurring when the water is driven into the air cavity are well resolved, and in agreement with the study of Xiang and Wang [74].

Finally, the pressure along the centreline of the water column without the cavity at time $t^{*}=0.8$ is plotted against the computational results of Xiang and Wang [74] in Fig. 13 and a good agreement is achieved. It has to be stressed that the expected negative pressure drop is not as pronounced as the study of Xiang and Wang [74] since their grid resolution was 

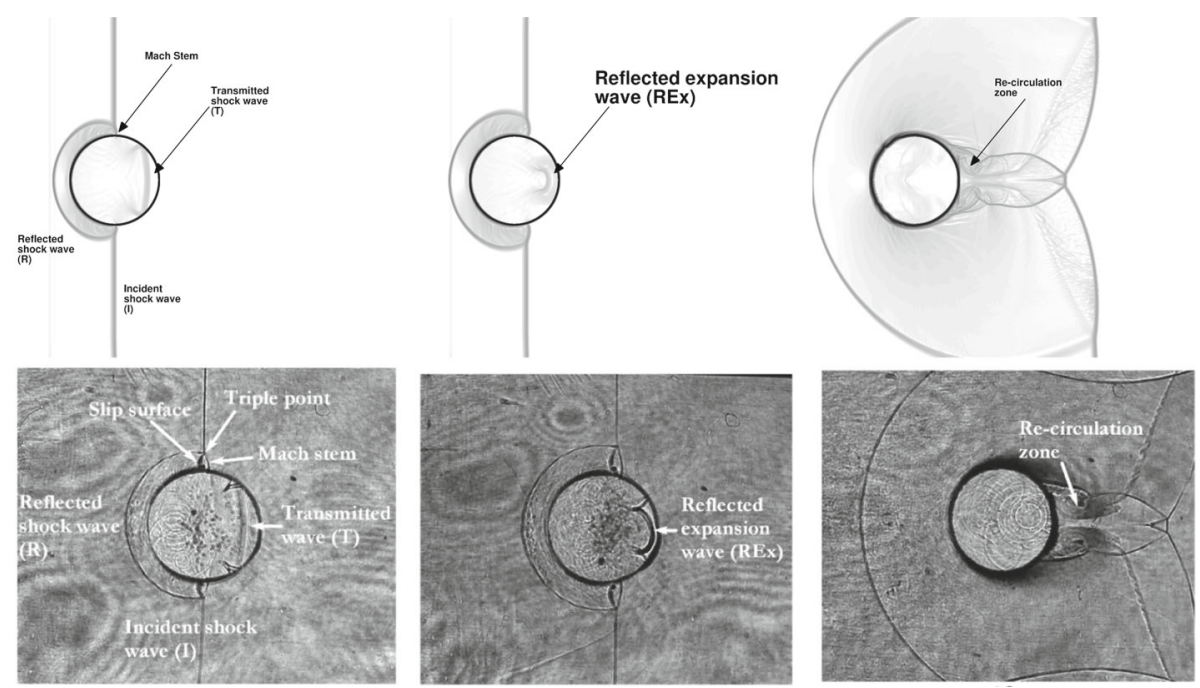

Fig. 11 Numerical Schlieren contours computed in this work (top) and obtained by experiments of Sembian et al. [48] (bottom) for the shock-wave interaction with a water column at $M=2.4$

finer with an edge length of of $e_{c} \approx D_{b} / 333$. However, the negative pressure is correctly captured due to the reflected expansion wave, which is attributed to the cohesive forces that hold liquids together and can withstand negative pressure [9,41]. For the water column with the air cavity, a time-history of the maximum pressure present in the computational domain is plotted against the computational results of Xiang and Wang [74] in Fig. 13, where it is evident that a good agreement is achieved although the peak pressure is slightly lower due to the lower grid resolution as expected. However the timing of the maximum peak pressure agrees with the results of [74], where the maximum peak pressure is seen at the time of impact of the transverse jet with the inner cavity wall downstream.

\subsection{Helium Bubble Shock Wave}

The interaction of a weak shockwave in air and a helium bubble is considered in 2D and 3D. Several variations of this test problem have been widely used [13,32,72] for assessing the performance of several techniques for multicomponent flow modelling, and is based on the experimental setup by Haas and Sturtevant [27]. A bubble of diameter $D_{b}=5 \mathrm{~cm}$, is placed within an air filled shock tube. The bubble consists of helium and air of $28 \%$ mass concentration. A shockwave moving from right to left as shown in Fig. 14 of the setup impacts the bubble contaminated by the surrounding air. The specific heats of 1.4 and 1.66 are used for air and helium, respectively, and the initial condition is given by:

$$
\left(a_{1} \rho_{1}, a_{2} \rho_{2}, u, v, p, a_{1}\right)= \begin{cases}(0.0,1.204,0,0,101325,0), & \text { for Pre-shock } \\ (0.0,1.658,-114.49,0,159060,0), & \text { for Post-shock } \\ (0.158,0.061,0,0,101325,0.95), & \text { for Bubble }\end{cases}
$$

The computation domain is discretised by a mixed-element unstructured mesh consisting of quadrilateral and triangular elements in 2D, and arbitrary hexahedrals and prism in 3D with 

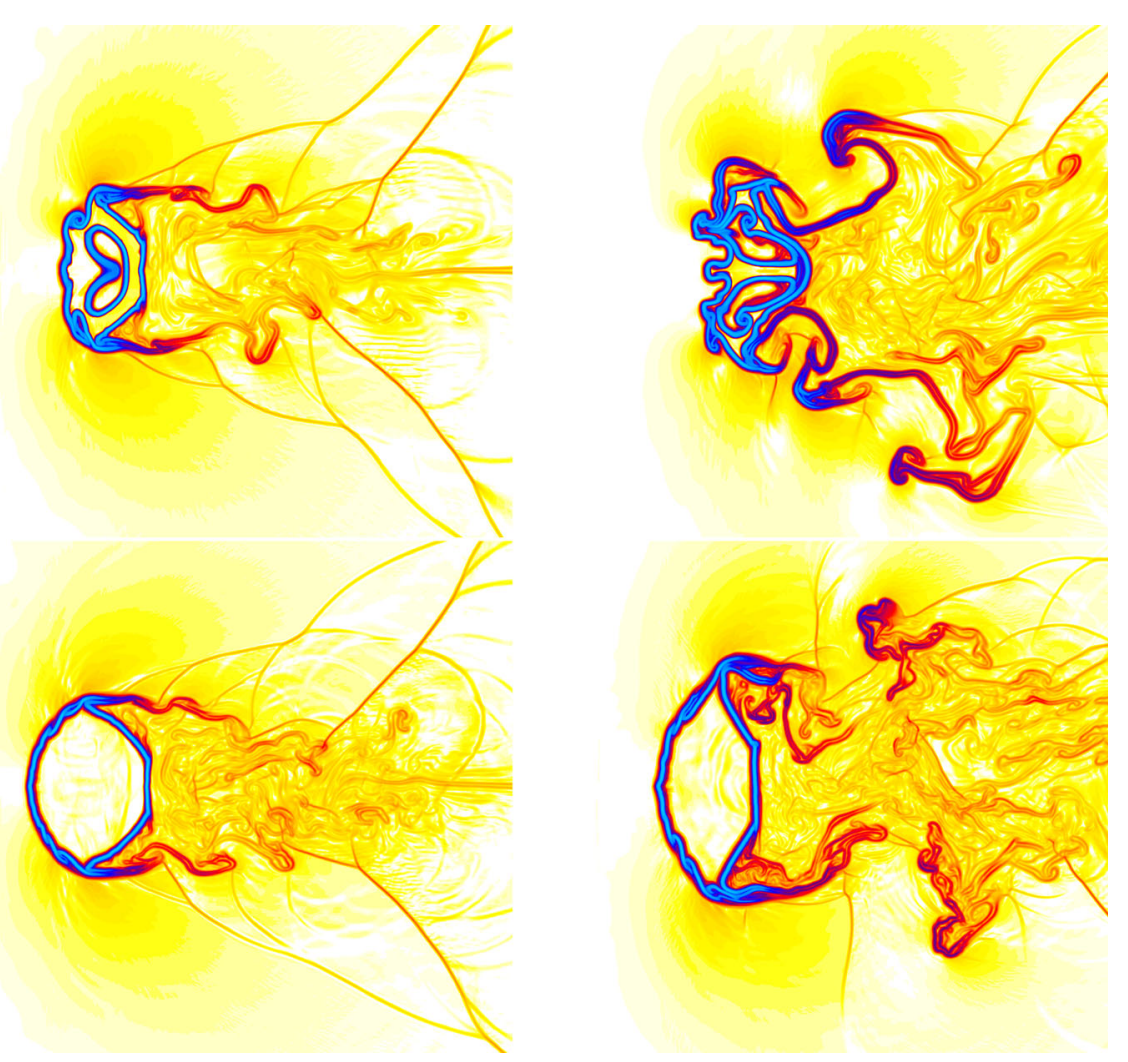

Fig. 12 Contour plots of density gradient magnitude of the computed solution of the shock wave interaction with a water column with a cavity (top) and without a cavity (bottom), at various instants $t^{*}=5.48, t^{*}=10.0$, (left to right). The flow separation for both variations as well as the transverse jet impact with the water cavity wall downstream (top) and the flattening of the water column downstream (bottom) can be noticed
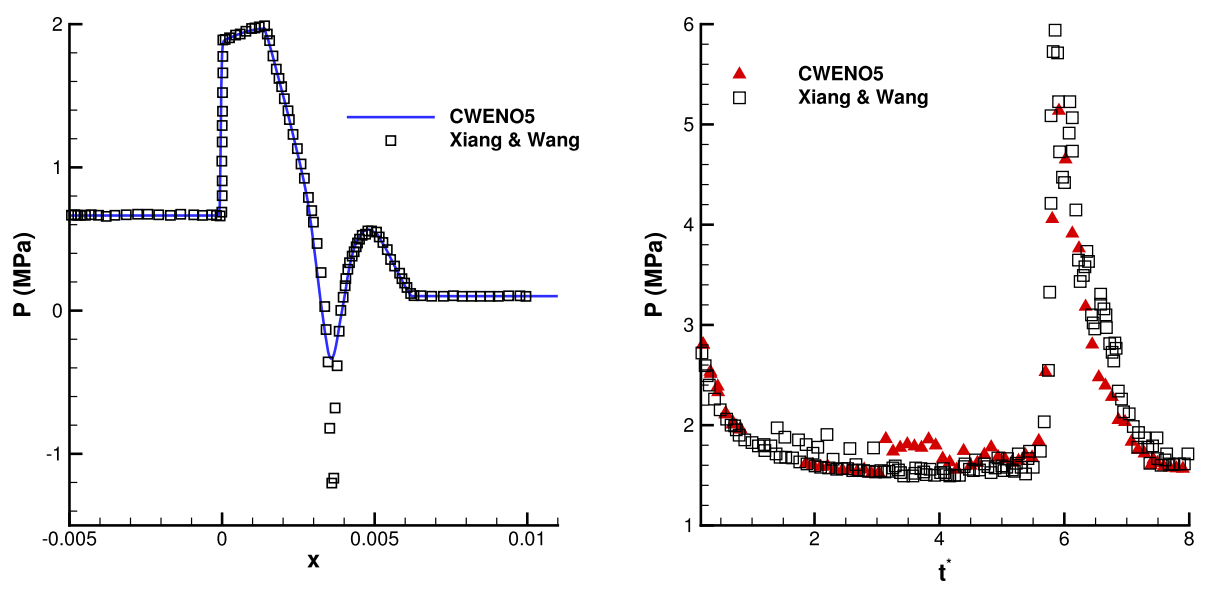

Fig. 13 Pressure distribution at the centreline of the water column at $t^{*}=0.8$ (left), and time evolution of the maximum pressure in the computational domain for the water column with an air cavity (right) and comparison with the results of Xiang and Wang [74] 


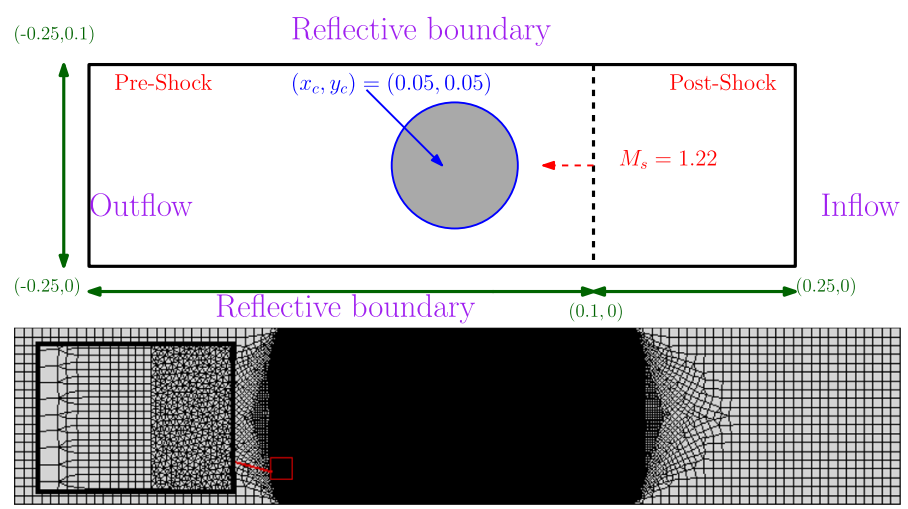

Fig. 14 Computational domain setup (top) and corresponding mesh for the helium bubble shock wave test problem

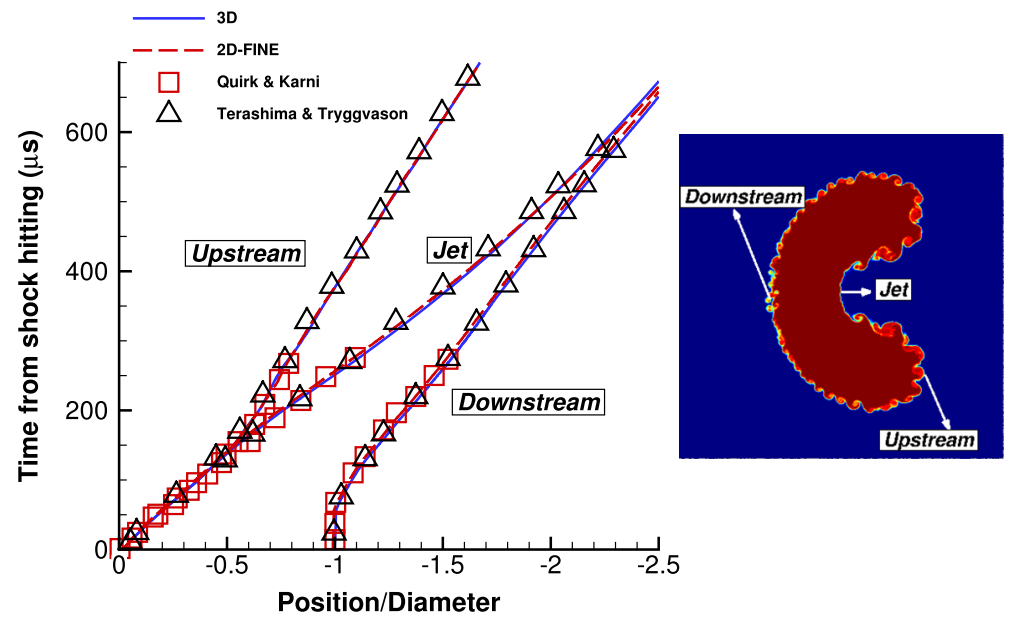

Fig. 15 Plot of the definition of the interfaces and of the evolution of the position of these interfaces using a CWENO5 scheme on the finest 2D mesh and 3D mesh. The evolution of the interfaces position is in good agreement with the results of Terashima and Tryggvason [51] and Quirk and Karni [44]

the shock-bubble interaction region being refined as shown in Fig. 14. A coarse, medium, and fine mesh are employed for $2 \mathrm{D}$ with an average element edge length in the shock bubble interaction zone of $e_{c} \approx D_{b} / 35, e_{m} \approx D_{b} / 120, e_{f} \approx D_{b} / 400$ respectively, and an edge length of $e_{c} \approx D_{b} / 50$ for the 3D mesh. A slip-wall boundary condition is used at the top and bottom boundaries of the domain, while inflow and outflow boundary conditions are prescribed at the right and left of the domain respectively. A CWENO5 scheme is employed and the simulation is run until $t=1000 \mu \mathrm{s}$.

From the results obtained as shown in Fig. 16, it can be noticed that the time evolution of the bubble is correctly captured including the formation of a jet and a vortex ring at late times, and is in agreement with the results obtained by $[13,32,72]$ qualitatively. Due to the high-order CWENO scheme employed, the Kelvin-Helmholtz instabilities that develop at the interface of the helium bubble are more pronounced as the 2D grid resolution is increased as shown in Fig. 17, whereas for the 3D setup the resolution employed is not sufficient to capture 


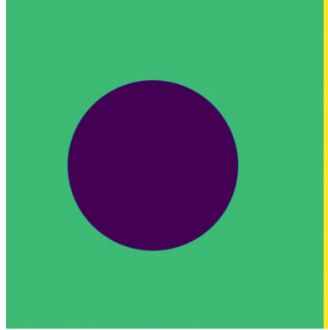

(a) $t=0$

(d) $t=403 \mu \mathrm{s}$

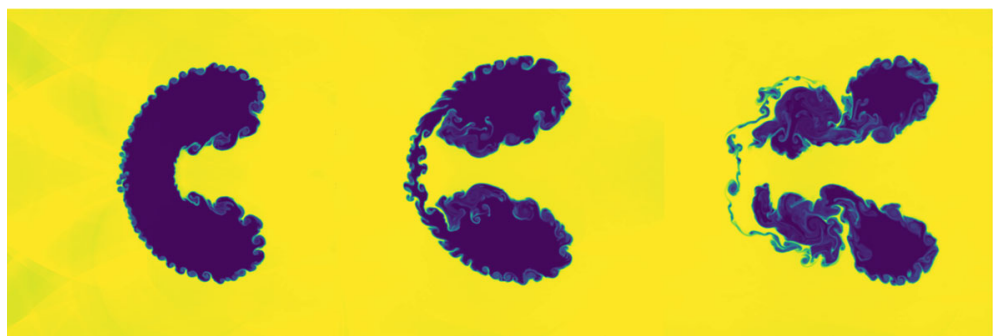

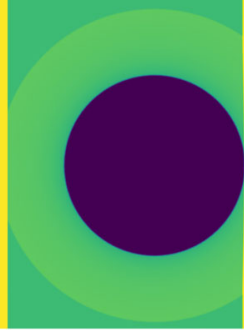

(b) $t=57 \mu \mathrm{s}$

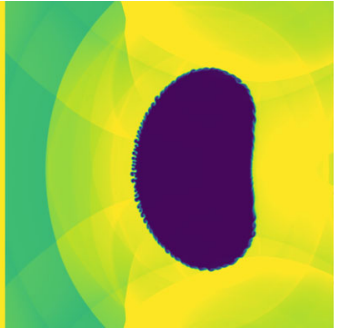

(c) $t=205 \mu \mathrm{s}$

(e) $t=603 \mu \mathrm{s}$

(f) $t=983 \mu \mathrm{s}$

Fig. 16 Density contour plots (24 equally distanced levels between 0.19 to 1.74 ) of the computed solution of the shockwave helium bubble interaction test problem at various instants using the finest mesh. As the shock wave passes the helium bubble, Kelvin-Helmholtz instabilities develop at the material interface that later on break down while resulting in an asymmetric solution profiles

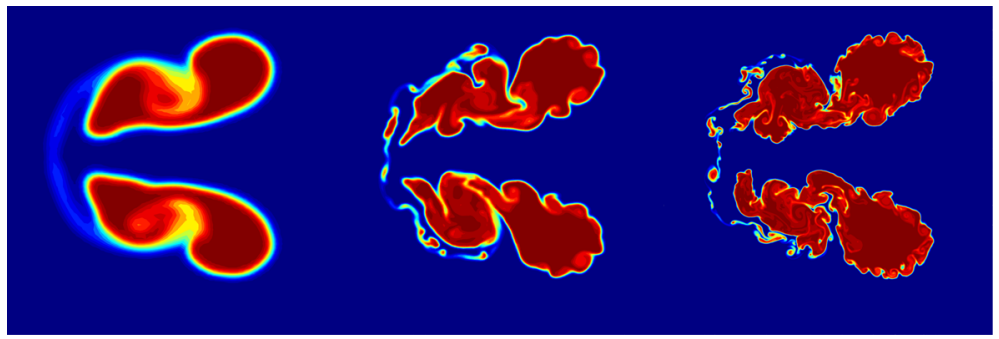

(a) coarse

(b) medium

(c) fine

Fig. 17 Contour plots of volume fraction ( 24 equally distanced levels between 0 to 1 ) of the computed solution of the shockwave helium bubble interaction test problem at $t=983 \mu \mathrm{s}$, at several mesh resolutions. More vortical structures are captured as the grid resolution is increased due to the absence of physical viscosity to diffuse them

these instabilities. as shown in as shown in Fig. 18. The symmetry of the computed solution is lost at late times for the 2D medium and fine meshes, indicative of multi-dimensional reconstruction nature of the framework employed and of the arbitrary unstructured elements in the refined shock-bubble interaction zone. As the bubble evolves, three distinct interfaces identified are the jet $(j i)$, the upstream $(u i)$ and the downstream (di) as shown in Fig. 15. From the space-time diagram of these distinct interface positions, the predicted locations are in good agreement with the reference results of Terashima and Tryggvason [51] and Quirk and Karni [44]. It needs to be highlighted that the results have been non-dimensionalised with respect to the diameter of the bubble at the time that the shock hits the bubble $[44,51,72]$. 


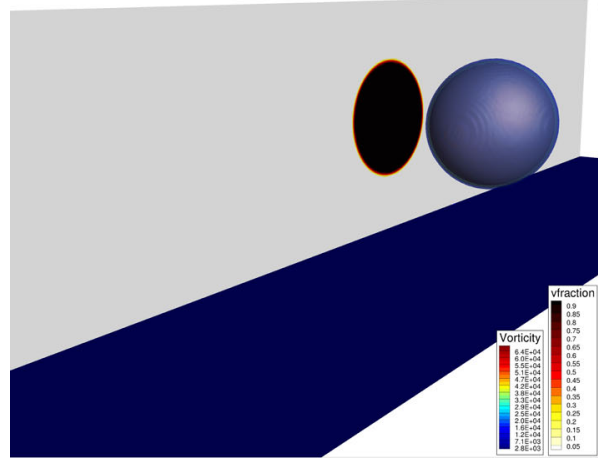

(a) $t=00 \mu \mathrm{s}$

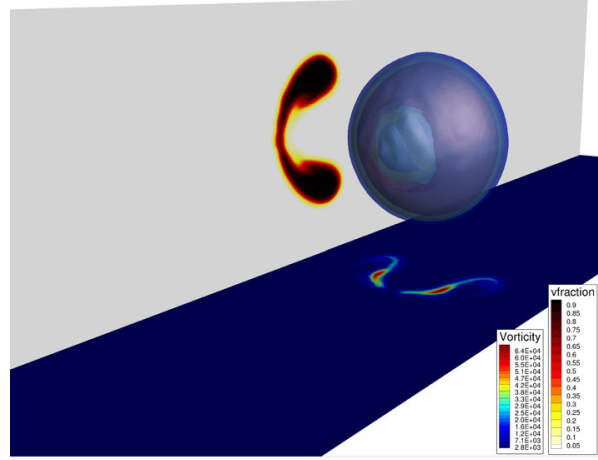

(c) $t=400 \mu \mathrm{s}$

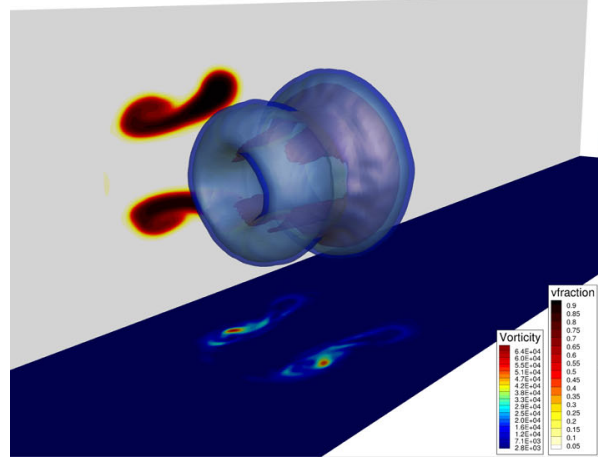

(e) $t=810 \mu \mathrm{s}$

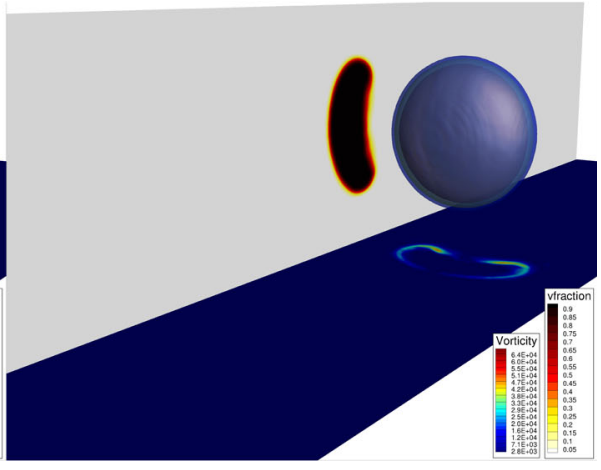

(b) $t=220 \mu \mathrm{s}$

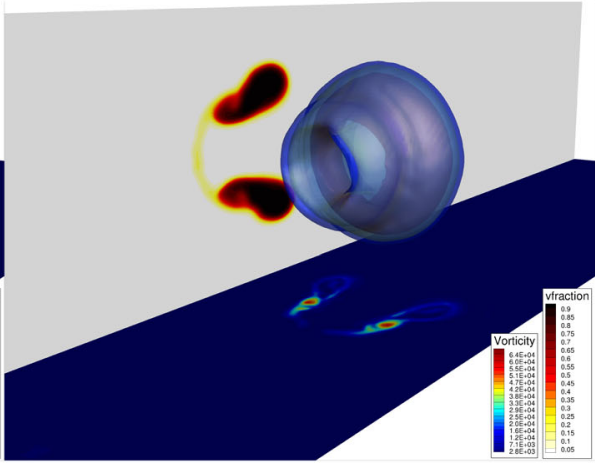

(d) $t=600 \mu \mathrm{s}$

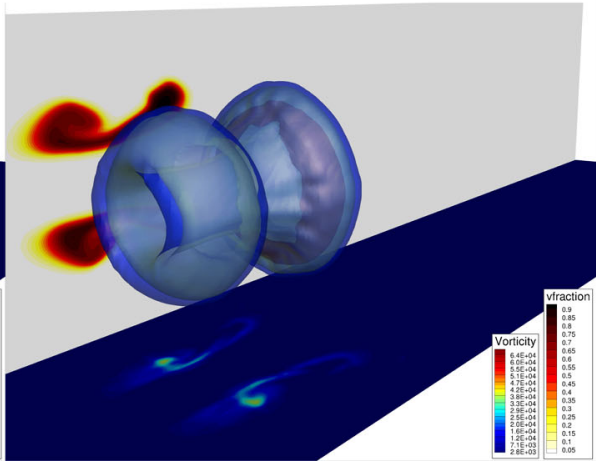

(f) $t=980 \mu \mathrm{s}$

Fig. 18 Contour plots of volume fraction, vorticity magnitude at the centre of the computational domain and isosurfaces of three levels of volume fraction $(0.25,0.5,0.9)$ of the computed solution of the shockwave 3D helium bubble interaction test problem at different instants 
Table 2 Time averaged velocities of several flow features of the shock-bubble interaction problem obtained with the present simulation and compared against the experimental results of Haas and Sturtevant [27], and the computational results of Coralic and Colonius [13]

\begin{tabular}{llll}
\hline Grid size & $u_{u i}(\mathrm{~m} / \mathrm{s})$ & $u_{j i}(\mathrm{~m} / \mathrm{s})$ & $u_{d i}(\mathrm{~m} / \mathrm{s})$ \\
\hline (2D) Coarse & 177.616 & 223.280 & 143.864 \\
(2D) Medium & 179.514 & 234.960 & 144.999 \\
(2D) Fine & 181.242 & 237.086 & 145.895 \\
3D & 178.402 & 228.375 & 144.197 \\
Coralic \& Colonius WENO5 [13] & 173 & 230 & 145 \\
Haas \& Sturtevant exp. [27] & $170 \pm 17$ & $230 \pm 23$ & $145 \pm 15$ \\
\hline
\end{tabular}

The time intervals that have been used to average the velocities are [10, 52] $\mu$ s for the upstrean interface, $[140,240] \mu s$ for the jet, and $[140,240] \mu s$ for the downstream interface. It can be noticed that all the obtained predictions are within the variations of the experiment, and in close agreement with the computational results of [13]

The results obtained in terms of the averaged velocities of the jet, upstream and downstream interface are in good agreement with the experimental results of Haas and Sturtevant [27], and the computational results of Coralic and Colonius [13] as shown in Table 2. Due to the resolution employed for the $3 \mathrm{D}$ setup of the problem, a slower merging of the jet and the downstream interface is seen compared to the $2 \mathrm{D}$ setup.

\section{Conclusions}

This paper extends to applicability of CWENO schemes on unstructured meshes to compressible multi-material flows. The schemes manage to achieve high-order of accuracy, resolve the material interfaces and finer structures while maintaining their essentially non-oscillatory character. Switching to primitive variable reconstruction was needed to remove the oscillations that appear across material interfaces. A series of stringent two- and three-dimensional test problems were used to verify the accuracy, robustness and computational efficiency of the schemes, and compared with analytical, experimental and computational results. Future development will concern the expansion of the CWENO schemes to the complete seven equation model of Baer-Nunziato's [6] and the unified hyperbolic formulation of GodunovPeshkov-Romenski (GPR model) [24,42].

Acknowledgements The authors acknowledge the computing time on the University of Cambridge Tier-2 national high-performance computing service Peta4 provided through the EPSRC grant EP/P020259/1, the computing time at HAWK (HLRS-DE) provided through the Project HOVE3 (EU-PRACE Grant ID: 823767), and the support of Innovate UK [Grant Number 263261]; and Airbus UK.

Data Availability The datasets generated during the current study are available at the Cranfield Online Research Data repository, [https://doi.org/10.17862/cranfield.rd.16447212.v1].

\section{Declarations}

Conflict of interest The authors declare that they have no conflict of interest.

Open Access This article is licensed under a Creative Commons Attribution 4.0 International License, which permits use, sharing, adaptation, distribution and reproduction in any medium or format, as long as you give 
appropriate credit to the original author(s) and the source, provide a link to the Creative Commons licence, and indicate if changes were made. The images or other third party material in this article are included in the article's Creative Commons licence, unless indicated otherwise in a credit line to the material. If material is not included in the article's Creative Commons licence and your intended use is not permitted by statutory regulation or exceeds the permitted use, you will need to obtain permission directly from the copyright holder. To view a copy of this licence, visit http://creativecommons.org/licenses/by/4.0/.

\section{References}

1. Allaire, G., Clerc, S., Kokh, S.: A five-equation model for the simulation of interfaces between compressible fluids. J. Comput. Phys. 181(2), 577-616 (2002). https://doi.org/10.1006/jcph.2002.7143

2. Antoniadis, A.F., Tsoutsanis, P., and Drikakis, D.: High-order schemes on mixed-element unstructured grids for aerodynamic flows. In: 42nd AIAA Fluid Dynamics Conference and Exhibit, vol. 2833 (2012). https://doi.org/10.2514/6.2012-2833

3. Antoniadis, A.F., Drikakis, D., Kokkinakis, I.W., Tsoutsanis, P., Ranam, Z.: High-order methods for hypersonic shock wave turbulent boundary layer interaction flow. In: 20th AIAA International Space Planes and Hypersonic Systems and Technologies Conference, vol. 3524 (2015). https://doi.org/10.2514/ 6.2015-3524

4. Antoniadis, A.F., Tsoutsanis, P., Drikakis, D.: Numerical accuracy in RANS computations of high-lift multi-element airfoil and aircraft configurations. In: 53rd AIAA Aerospace Sciences Meeting, vol. 0317 (2015). https://doi.org/10.2514/6.2015-0317

5. Antoniadis, A.F., Tsoutsanis, P., Kokkinakis, I., Rana, Z., Drikakis, D.: Azure: an advanced CFD software suite based on high-resolution and high-order methods. In: 53rd AIAA Aerospace Sciences Meeting, vol. 0813 (2015). https://doi.org/10.2514/6.2015-0813

6. Baer, M., Nunziato, J.: A two-phase mixture theory for the deflagration-to-detonation transition (DDT) in reactive granular materials. Int. J. Multiph. Flow 12, 861-889 (1986)

7. Bakhvalov, P., Kozubskaya, T.: EBR-WENO scheme for solving gas dynamics problems with discontinuities on unstructured meshes. Comput. Fluids 157, 312-324 (2017)

8. Barton, P.T.: An interface-capturing Godunov method for the simulation of compressible solid-fluid problems. J. Comput. Phys. 390, 25-50 (2019). https://doi.org/10.1016/j.jcp.2019.03.044

9. Caupin, F.: Liquid-vapor interface,cavitation, and the phase diagram of water. Phys. Rev. E 71, 051605 (2005). https://doi.org/10.1103/PhysRevE.71.051605

10. Cheng, L., Deng, X., Xie, B., Jiang, Y., Xiao, F.: Low-dissipation BVD schemes for single and multi-phase compressible flows on unstructured grids. J. Comput. Phys. (2021). https://doi.org/10.1016/j.jcp.2020. 110088

11. Chiapolino, A., Saurel, R., Nkonga, B.: Sharpening diffuse interfaces with compressible fluids on unstructured meshes. J. Comput. Phys. 340, 389-417 (2017). https://doi.org/10.1016/j.jcp.2017.03.042

12. Cocchi, J.P., Saurel, R., Loraud, J.C.: Treatment of interface problems with Godunov-type schemes. Shock Waves 5(1), 347-357 (1996)

13. Coralic, V., Colonius, T.: Finite-volume WENO scheme for viscous compressible multicomponent flows. J. Comput. Phys. 274, 95-121 (2014). https://doi.org/10.1016/j.jcp.2014.06.003

14. Cutforth, M., Barton, P.T., Nikiforakis, N.: An efficient moment-of-fluid interface tracking method. Comput. Fluids (2021). https://doi.org/10.1016/j.compfluid.2021.104964

15. Diot, S., Clain, S., Loubére, R.: Improved detection criteria for the multi-dimensional optimal order detection (MOOD) on unstructured meshes with very high-order polynomials. Comput. Fluids 64, 43-63 (2012). https://doi.org/10.1016/j.compfluid.2012.05.004

16. Dumbser, M., Kaser, M.: Arbitrary high order non-oscillatory finite volume schemes on unstructured meshes for linear hyperbolic systems. J. Comput. Phys. 221(2), 693-723 (2007)

17. Dumbser, M., Kaser, M., Titarev, V.A., Toro, E.F.: Quadrature-free non-oscillatory finite volume schemes on unstructured meshes for nonlinear hyperbolic systems. J. Comput. Phys. 226(1), 204-243 (2007)

18. Dumbser, M., Hidalgo, A., Castro, M., Parés, C., Toro, E.F.: Force schemes on unstructured meshes II: non-conservative hyperbolic systems. Comput. Methods Appl. Mech. Eng. 199(9-12), 625-647 (2010). https://doi.org/10.1016/j.cma.2009.10.016

19. Dumbser, M., Boscheri, W., Semplice, M., Russo, G.: Central weighted ENO schemes for hyperbolic conservation laws on fixed and moving unstructured meshes. SIAM J. Sci. Comput. 39(6), A2564-A2591 (2017). https://doi.org/10.1137/17M1111036 
20. Farmakis, P.S., Tsoutsanis, P., Nogueira, X.: WENO schemes on unstructured meshes using a relaxed a posteriori MOOD limiting approach. Comput. Methods Appl. Mech. Eng. (2020). https://doi.org/10. 1016/j.cma.2020.112921

21. Faucher, V., Bulik, M., Galon, P.: Updated VOFIRE algorithm for fast fluid-structure transient dynamics with multi-component stiffened gas flows implementing anti-dissipation on unstructured grids. J. Fluids Struct. 74, 64-89 (2017). https://doi.org/10.1016/j.jfluidstructs.2017.07.001

22. Favrie, N., Gavrilyuk, S.L.: Diffuse interface model for compressible fluid-compressible elastic-plastic solid interaction. J. Comput. Phys. 231(7), 2695-2723 (2012). https://doi.org/10.1016/j.jcp.2011.11.027

23. Favrie, N., Gavrilyuk, S.L., Saurel, R.: Solid-fluid diffuse interface model in cases of extreme deformations. J. Comput. Phys. 228(16), 6037-6077 (2009). https://doi.org/10.1016/j.jcp.2009.05.015

24. Godunov, S.K., Romenski, E.: Nonstationary equations of nonlinear elasticity theory in Eulerian coordinates. J. Appl. Mech. Tech. Phys. 13, 868-884 (1972). https://doi.org/10.1007/BF01200547

25. Goncalves, E., Hoarau, Y., Zeidan, D.: Simulation of shock-induced bubble collapse using a four-equation model. Shock Waves 29(1), 221-234 (2019). https://doi.org/10.1007/s00193-018-0809-1

26. Gottlieb, S., Shu, C.-W.: Total variation diminishing Runge-Kutta schemes. Math. Comput. 67(221), 73-85 (1998). https://doi.org/10.1090/S0025-5718-98-00913-2

27. Haas, J.F., Sturtevant, B.: Interaction of weak shock waves with cylindrical and spherical gas inhomogeneities. J. Fluid Mech. 181, 41-76 (1987). https://doi.org/10.1017/S0022112087002003

28. Hirt, C.W., Nichols, B.D.: Volume of fluid (VOF) method for the dynamics of free boundaries. J. Comput. Phys. 39(1), 201-225 (1981). https://doi.org/10.1016/0021-9991(81)90145-5

29. Jackson, H., Nikiforakis, N.: A unified Eulerian framework for multimaterial continuum mechanics. J. Comput. Phys. (2020). https://doi.org/10.1016/j.jcp.2019.109022

30. Jalali, A., Ollivier-Gooch, C.: Higher-order finite volume solution reconstruction on highly anisotropic meshes. In: 21st AIAA Computational Fluid Dynamics Conference (2013)

31. Johnsen, E.: On the treatment of contact discontinuities using WENO schemes. J. Comput. Phys. 230(24), 8665-8668 (2011). https://doi.org/10.1016/j.jcp.2011.08.017

32. Johnsen, E., Colonius, T.: Implementation of WENO schemes in compressible multicomponent flow problems. J. Comput. Phys. 219(2), 715-732 (2006). https://doi.org/10.1016/j.jcp.2006.04.018

33. Johnsen, E., Ham, F.: Preventing numerical errors generated by interface-capturing schemes in compressible multi-material flows. J. Comput. Phys. 231(17), 5705-5717 (2012). https://doi.org/10.1016/j.jcp. 2012.04.048

34. Johnsen, E., Larsson, J., Bhagatwala, A.V., Cabot, W.H., Moin, P., Olson, B.J., Rawat, P.S., Shankar, S.K., Sjogreen, B., Yee, H.C., Zhong, X., Lele, S.K.: Assessment of high-resolution methods for numerical simulations of compressible turbulence with shock waves. J. Comput. Phys. 229(4), 1213-1237 (2010). https://doi.org/10.1016/j.jcp.2009.10.028

35. Kapila, A., Menikoff, R., Bdzil, J.B., Son, S.F., Stewart, D.S.: Two-phase modeling of deflagration-todetonation transition in granular materials: reduced equations. Phys. Fluids 13, 3002-3024 (2001)

36. Kokkinakis, I.W., Drikakis, D., Youngs, D.L.: Vortex morphology in Richtmyer-Meshkov-induced turbulent mixing. Physica D: Nonlinear Phenom. (2020). https://doi.org/10.1016/j.physd.2020.132459

37. Meng, J.C., Colonius, T.: Numerical simulations of the early stages of high-speed droplet breakup. Shock Waves 25, 399-414 (2015). https://doi.org/10.1016/j.jcp.2014.06.003

38. Ndanou, S., Favrie, N., Gavrilyuk, S.: Multi-solid and multi-fluid diffuse interface model: applications to dynamic fracture and fragmentation. J. Comput. Phys. 295, 523-555 (2015). https://doi.org/10.1016/ j.jcp.2015.04.024

39. Nourgaliev, R.R., Dinh, T.N., Theofanous, T.G.: Adaptive characteristics-based matching for compressible multifluid dynamics. J. Comput. Phys. 213(2), 500-529 (2006). https://doi.org/10.1016/j.jcp.2009.10.028

40. Osher, S., Sethian, J.A.: Fronts propagating with curvature-dependent speed: algorithms based on Hamilton-Jacobi formulations. J. Comput. Phys. 79(1), 12-49 (1988). https://doi.org/10.1016/00219991(88)90002-2

41. Pattersen, M.S., Balibar, S., Maris, H.J.: Experimental investigation in superfluid He. Phys. Rev. B 49, 12062-12070 (1994)

42. Peshkov, I., Romenski, E.: A hyperbolic model for viscous Newtonian flows. Contin. Mech. Thermodyn. 28, 85-104 (2016). https://doi.org/10.1007/s00161-014-0401-6

43. Price, M.A., Nguyen, V.-T., Hassan, O., Morgan, K.: A method for compressible multimaterial flows with condensed phase explosive detonation and airblast on unstructured grids. Comput. Fluids 111, 76-90 (2015). https://doi.org/10.1016/j.compfluid.2015.01.006

44. Quirk, J.J., Karni, S.: On the dynamics of a shock-bubble interaction. J. Fluid Mech. 318, 129-163 (1996). https://doi.org/10.1017/S0022112096007069

45. Rayleigh, L.: On the theory of surface forces.-II compressible fluids. Philos. Mag. 33, 209-220 (1892) 
46. Ricci, F., Silva, P.A.S.F., Tsoutsanis, P., Antoniadis, A.F.: Hovering rotor solutions by high-order methods on unstructured grids. Aerosp. Sci. Technol. (2020). https://doi.org/10.1016/j.ast.2019.105648

47. Schmidmayer, K., Bryngelson, S.H., Colonius, T.: An assessment of multicomponent flow models and interface capturing schemes for spherical bubble dynamics. J. Comput. Phys. (2020). https://doi.org/10. 1016/j.jcp.2019.109080

48. Sembian, S., Liverts, M., Tillmark, N., Apazidis, N.: Plane shock wave interaction with a cylindrical water column. Phys. Fluids (2016). https://doi.org/10.1063/1.4948274

49. Silva, P.A.S.F., Tsoutsanis, P., Antoniadis, A.F.: Simple multiple reference frame for high-order solution of hovering rotors with and without ground effect. Aerosp. Sci. Technol. (2021). https://doi.org/10.1016/ j.ast.2021.106518

50. Simmonds, N., Tsoutsanis, P., Antoniadis, A.F., Jenkins, K.W., Gaylard, A.: Low-Mach number treatment for finite-volume schemes on unstructured meshes. Appl. Math. Comput. 336, 368-393 (2018)

51. Terashima, H., Tryggvason, G.: A front-tracking/ghost-fluid method for fluid interfaces in compressible flows. J. Comput. Phys. 228(11), $4012-4037$ (2009). https://doi.org/10.1016/j.jcp.2009.02.023

52. Tillyaeva, N.I.: Generalisation of modified Godunov scheme to arbitrary irregular meshes. Trans. Central Aerohydrodyn. Inst. 17(2), 18-26 (1986)

53. Titarev, V.A., Tsoutsanis, P., Drikakis, D.: WENO schemes for mixed-element unstructured meshes. Commun. Comput. Phys. 8(3), 585-609 (2010)

54. Toro, E.F., Spruce, M., Speares, W.: Restoration of the contact surface in the HLL-Riemann solver. Shock Waves 4(1), 25-34 (1994)

55. Tryggvason, G., Bunner, B., Esmaeeli, A., Juric, D., Al-Rawahi, N., Tauber, W., Han, J., Nas, S., Jan, Y.-J.: A front-tracking method for the computations of multiphase flow. J. Comput. Phys. 169(2), 708-759 (2001). https://doi.org/10.1006/jcph.2001.6726

56. Tsoutsanis, P.: KNL performance comparison UCNS3D. ARCHER performance report, pp. 157-170 (2017). www.archer.ac.uk/community/benchmarks/archer-knl/KNLperfUCNS3D.pdf

57. Tsoutsanis, P.: Extended bounds limiter for high-order finite-volume schemes on unstructured meshes. J. Comput. Phys. 362, 69-94 (2018)

58. Tsoutsanis, P.: Stencil selection algorithms for WENO schemes on unstructured meshes. J. Comput. Phys.: X (2019). https://doi.org/10.1016/j.jcpx.2019.100037

59. Tsoutsanis, P., Drikakis, D.: A high-order finite-volume method for atmospheric flows on unstructured grids. J. Coupled Syst. Multiscale Dyn. 4, 170-186 (2016). https://doi.org/10.1166/jcsmd.2016.1104

60. Tsoutsanis, P., Drikakis, D.: Addressing the challenges of implementation of high-order finite-volume schemes for atmospheric dynamics on unstructured meshes. In: ECCOMAS Congress 2016, Crete, Greece (2016). https://doi.org/10.7712/100016.1846.8406

61. Tsoutsanis, P., Dumbser, M.: Arbitrary high order central non-oscillatory schemes on mixed-element unstructured meshes. Comput. Fluids (2021). https://doi.org/10.1016/j.compfluid.2021.104961

62. Tsoutsanis, P., Srinivasan, H.: Adaptive mesh refinement techniques for high-order finite-volume WENO schemes. In: ECCOMAS Congress 2016, Crete, Greece (2016). https://doi.org/10.7712/100016.2003. 8544

63. Tsoutsanis, P., Titarev, V.A., Drikakis, D.: WENO schemes on arbitrary mixed-element unstructured meshes in three space dimensions. J. Comput. Phys. 230(4), 1585-1601 (2011)

64. Tsoutsanis, P., Antoniadis, A.F., Drikakis, D.: WENO schemes on arbitrary unstructured meshes for laminar, transitional and turbulent flows. J. Comput. Phys. 256, 254-276 (2014)

65. Tsoutsanis, P., Kokkinakis, I.W., Konozsy, L., Drikakis, D., Williams, R.J.R., Youngs, D.L.: Comparison of structured- and unstructured-grid, compressible and incompressible methods using the vortex pairing problem. Comput. Methods Appl. Mech. Eng. 293, 207-231 (2015). https://doi.org/10.1016/j.cma.2015. 04.010

66. Tsoutsanis, P., Simmonds, N., Gaylard, A.: Implementation of a low-Mach number modification for highorder finite-volume schemes for arbitrary hybrid unstructured meshes. In: ECCOMAS Congress 2016, Crete, Greece (2016). https://doi.org/10.7712/100016.2004.8545

67. Tsoutsanis, P., Antoniadis, A.F., Jenkins, K.W.: Improvement of the computational performance of a parallel unstructured WENO finite volume CFD code for implicit large eddy simulation. Comput. Fluids 173, 157-170 (2018). https://doi.org/10.1016/j.compfluid.2018.03.012

68. UCNS3D CFD code. http://www.ucns3d.com. Accessed: 10 March 2021

69. van der Waals, J.D.: Thermodynamische theorie der capillariteit in de onderstelling van continue dichtheidsverandering. Verhandel. Konink. Akad. Weten. Amsterdam (Sect1) (Philos. Mag.) 1, 56 (1893)

70. Wallis, T., Barton, P.T., Nikiforakis, N.: A flux-enriched Godunov method for multi-material problems with interface slide and void opening. J. Comput. Phys. (2021). https://doi.org/10.1016/j.jcp.2021.110499

71. Wallis, T., Barton, P.T., Nikiforakis, N.: A diffuse interface model of reactive-fluids and solid-dynamics. Comput. Struct. (2021). https://doi.org/10.1016/j.compstruc.2021.106578 
72. Wang, Q., Deiterding, R., Pan, J., Ren, Y.-X.: Consistent high resolution interface-capturing finite volume method for compressible multi-material flows. Comput. Fluids (2020). https://doi.org/10.1016/j. compfluid.2020.104518

73. Wong, M.L., Lele, S.K.: High-order localized dissipation weighted compact nonlinear scheme for shockand interface-capturing in compressible flows. J. Comput. Phys. 339, 179-209 (2017). https://doi.org/10. 1016/j.jcp.2017.03.008

74. Xiang, G., Wang, B.: Numerical study of a planar shock-interacting with a cylindrical water column embedded with an air cavity. J. Fluid Mech. 825, 825-852 (2017). https://doi.org/10.1017/jfm.2017.403

75. Zhu, J., Shu, C.-W.: A new type of third-order finite volume multi-resolution WENO schemes on tetrahedral meshes. J. Comput. Phys. 406, 109212 (2020)

Publisher's Note Springer Nature remains neutral with regard to jurisdictional claims in published maps and institutional affiliations. 